\title{
¿On the Formation Mechanism of the Seasonal Persistence Barrier
}

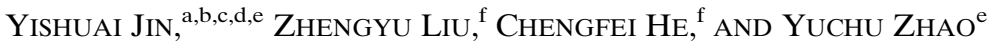

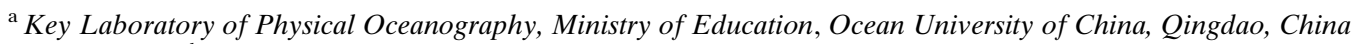 \\ ${ }^{\mathrm{b}}$ Institute for Advanced Ocean Study, Ocean University of China, Qingdao, China \\ ${ }^{\mathrm{c}}$ Frontiers Science Center for Deep Ocean Multispheres and Earth System, Ocean University of China, Qingdao, China \\ ${ }^{\mathrm{d}}$ Open Studio for Ocean-Climate-Isotope Modeling, Pilot National Laboratory for Marine Science and Technology \\ (Qingdao), Qingdao, China \\ ${ }^{\mathrm{e}}$ Department of Atmospheric and Oceanic Sciences, Peking University, Beijing, China \\ ${ }^{\mathrm{f}}$ Atmospheric Science Program, Department of Geography, The Ohio State University, Columbus, Ohio
}

(Manuscript received 5 July 2019, in final form 3 October 2020)

\begin{abstract}
The mechanism of the seasonal persistence barrier (SPB) is studied in the framework of an autoregressive (AR) model. In contrast to the seasonal variance, whose minimum is modulated mainly by the minimum growth rate or noise forcing, the SPB is caused primarily by the declining growth rate or increasing noise forcing, instead of the minimum/ maximum of the growth rate or noise forcing. In other words, the SPB is caused by the declining signal-to-noise ratio (SNR) rather than the weakest SNR. In a weakly damped system, the phase of the SPB is delayed from that of declining SNR by about a season. The mechanism is further applied to explain the observed SST variability in the tropical and North Pacific. For the tropical Pacific, the spring SPB could be caused by the decreasing growth rate from September to March and weak annual mean damping rate, instead of the minimum growth rate in spring. Over the North Pacific, the increasing noise forcing from March to June may lead to the summer SPB. Our mechanism provides a null hypothesis for understanding the SPB of climate variability.
\end{abstract}

KEYWORDS: Numerical analysis/modeling; Seasonal forecasting; Climate variability; Seasonal cycle

\section{Introduction}

The seasonal persistence barrier (SPB) is well known in the study of El Niño-Southern Oscillation (ENSO) prediction. It has been noticed in the observations of the tropical Pacific sea surface temperature anomalies (SSTA) (e.g., Niño-3.4, $5^{\circ} \mathrm{S}-$ $5^{\circ} \mathrm{N}, 170^{\circ}-120^{\circ} \mathrm{W}$; Ren et al. 2016) variability and the following variability in rainfall (Walker and Bliss 1932; Wright 1979) and sea level pressure (Troup 1965; Webster and Yang 1992). This SPB for ENSO features a band of maximum decline of monthly autocorrelation at a fixed phase (or month, typically around May-June), as seen from the monthly autocorrelation of SSTA variability over central-eastern Pacific and its lag gradient (e.g., Ren et al. 2016; Liu et al. 2019). This maximum decline of persistence of fixed phase shows that, regardless of the initial month of forecast, a damped persistence forecast loses its predictability most rapidly in the following May, forming the so-called the spring SPB of ENSO. Recent studies have also shown similar SPB features in many other areas such as the western North Pacific reemergence region $\left(38^{\circ}-42^{\circ} \mathrm{N}, 160^{\circ} \mathrm{E}-\right.$ 180 ; Alexander et al. 1999) (Zhao et al. 2012) and Southern Ocean (Liu et al. 2019), where SSTA variability also exhibits a band of maximum persistence decline at the fixed calendar

\footnotetext{
¿ Denotes content that is immediately available upon publication as open access.
}

Corresponding authors: Yishuai Jin, jinyishuai@126.com; Zhengyu Liu, liu.7022@osu.edu month, or phase locked to the specific month/season. In spite of these studies, it has remained unclear what exactly is the cause of the SPB, and in particular what factor determines the phase of a SPB.

In the theories where ENSO is considered as a self-sustained interannual oscillator in the tropical Pacific (Chen et al. 2004; Yeh et al. 2004; Mu et al. 2007), the growth of initial error is responsible for the predictability and SPB of ENSO (Zebiak 1989; Goswami and Shukla 1991). Chen and Cane (2008) found that model initialization and data assimilation were important for ENSO SPB. In the analysis of ENSO prediction in 10 different ocean-atmosphere coupled general circulation models (CGCMs), Jin et al. (2008) suggested that the seasonal cycle of initial conditions has an overwhelming role in ENSO SPB.

On the other hand, ENSO has also been considered in a damped noise-driven framework (Penland and Sardeshmukh 1995; Thompson and Battisti 2000; Kim and Jin 2011). Here the spring SPB phenomenon has been studied extensively using the cyclostationary autoregressive (AR) model (Torrence and Webster 1998; Moon and Wettlaufer 2017), as well as theoretical oscillator models with a seasonal cycle (Stein et al. 2010; Levine and McPhaden 2015). In these simple models, the seasonal growth rate is considered to play a significant role in ENSO spring SPB. Liu et al. (2019) derived a simple analytical solution for the SPB as the asymptotic solution of the damped persistence model with seasonal growth rate and noise forcing (i.e., a Langevin equation of seasonally varying coefficients). This solution showed explicitly that the amplitude of the seasonal cycle, either in growth rate or noise forcing, has to exceed a threshold to generate a SPB. This theory also 
suggests a general phase relationship between the seasonally varying variance and lag-1 autocorrelation coefficient (persistence) (Jin et al. 2019a).

Previous studies have also discussed the formation mechanism of the SPB, especially in the context of ENSO. Xue et al. (1994) attributed the spring SPB of ENSO to the small variance in spring. Webster (1995) suggested that the tropical coupled system was at its most stable state during the boreal spring with the minimum signal-to-noise ratio (SNR). Using a cyclostationary AR model, Torrence and Webster (1998) further concluded that in the absence of seasonal noise forcing, the spring SPB of ENSO was caused by the low growth rate in spring. These studies may lead to the impression that it is the minimum growth rate in spring that causes the lowest variance and, in turn, SNR in spring, which then leads to the SPB in spring. In this view, SPB is caused by, and then concurs with, the minimum growth rate or variance. This, however, does not seem to be always true in the analytical solution of Liu et al. (2019). For example, in the case of seasonal damping rate (i.e., negative growth rate), the solution of Liu et al. (2019) implies that the phase of the SPB changes, qualitatively, with both the annual mean damping rate and the amplitude of the annual cycle (parameters $b$ and $A$, respectively; see their Fig. 3b). As the annual mean damping rate (parameter $b$ ) diminishes, the response of system tends to be delayed, with the maximum delay of $90^{\circ}$ (three months), and the timing of SPB is also shifted later. As the amplitude of seasonal cycle (parameter $A$ ) increases, the timing of SPB will also be delayed. As such, the SPB can even occur in the maximum growth rate/variance for certain range of $b$ and $A$ [Fig. 3a in Liu et al. (2019)]. This implies that, in general, the minimum growth rate/variance is unlikely to be the major cause of the SPB.

In spite of its qualitative utility, the analytical solution of Liu et al. (2019) is difficult to use to study the exact mechanism for the phase of the SPB, because it uses a smooth harmonic form of the seasonal cycle of growth rate or noise forcing. Therefore, the nonharmonic annual cycle will be needed to further answer the question of the formation mechanism of SPB, especially for the relationship between the growth rate/noise forcing and the SPB.

This paper is an attempt to further understand the formation mechanism of the SPB, especially its phase, beyond the theory of Liu et al. (2019). Here, we will use an idealized nonharmonic annual cycle to pinpoint the cause for the formation of the SPB.
Our major conclusion is that the SPB is caused by the decreasing growth rate or increasing noise forcing, instead of the minimum growth rate or the maximum noise forcing (weakest SNR). An application of our mechanism to the observation shows that the SPB for ENSO is forced by the decreased growth rate from September to March and is then further delayed by the weak annual mean damping whereas the summer SPB in North Pacific is caused by the increasing noise forcing from March to May.

The paper is organized as follows. Section 2 describes the models used here. The SPBs in the idealized cases forced by growth rate and noise forcing are shown in sections 3 and 4, respectively. The application of our mechanism to the Pacific is carried out in section 5. Finally, a summary and discussion will be addressed in section 6 .

\section{Models}

\section{a. AR model}

In this study, we will explore the SPB numerically in the AR(1) model (e.g., Torrence and Webster 1998; Lu and Liu 2018) instead of the stochastic climate model (Hasselmann 1976). This is because we will consider various forms of the seasonal cycle of growth rate/noise forcing, which could not be studied easily in analytical solutions in a stochastic climate model as in Liu et al. (2019). The cyclostationary AR(1) model with the seasonal cycle can be described as

$$
X_{t+1}=a_{m} X_{t}+N_{t+1}
$$

where $X$ represents the variable (e.g., SSTA) and the subscript $t$ is the time measured in months starting from year zero; $a_{m}$ is the growth rate at calendar month $m ; N_{t}$ is the random noise drawn from the 12 Gaussian white noise of zero mean and variances $\sigma_{\mathrm{Nm}}^{2}$ (Torrence and Webster 1998; Lu and Liu 2018; Liu et al. 2019; Jin et al. 2019a). We note that in the observations, noise forcing may not be a pure white noise, especially for the tropical Pacific (Levine and McPhaden 2015). For the SPB phenomenon, however, the types of noise used in this study do not play a significant role (more details can be seen in appendix A).

The variance $\sigma_{m}^{2}$ and autocorrelation function $r_{m}^{k}(k$ being the lag month from the initial calendar month $m$ ) of Eq. (2.1) can be derived analytically, following Torrence and Webster (1998), as

$$
\sigma_{m}^{2}=\frac{\sigma_{N m}^{2}+\sigma_{N m-1}^{2} a_{m-1}^{2}+\sigma_{N m-2}^{2} a_{m-1}^{2} a_{m-2}^{2}+\cdots+\sigma_{N m-11}^{2} \prod_{j=1}^{11} a_{m-j}^{2}}{1-\prod_{j=1}^{12} a_{j}} .
$$

The physical meaning of Eq. (2.2) is that the variance in a specific month $m$ is dominantly caused by the noise forcing of this month, and second influenced by the noise forcing of previous month $m-j$ ( $j$ from 1 to 12 ) damped by the growth rate of months from $m-l\left(a_{m-1}\right)$ to $m-j\left(a_{m-j}\right)$ as the weight coefficient.

The autocorrelation function $r_{m}^{k}$ is described as

$$
r_{m}^{k}=\frac{\sigma_{m}}{\sigma_{m+k}} \prod_{j=0}^{k-1} a_{m+j}
$$

All the figures for AR(1) model are from the analytical solutions of Eqs. (2.2) and (2.3).

The SNR can be defined as in Torrence and Webster (1998) as 


$$
\mathrm{SNR}_{m}=\frac{\sigma_{m}^{2}-\sigma_{N m}^{2}}{\sigma_{N m}^{2}}
$$

\section{b. The recharge oscillator model}

To test whether the AR model has the ability to explain observational ENSO spring SPB, we also introduce the recharge oscillator model (Stein et al. 2010). This recharge oscillator captures the dynamic relationship between equatorial Pacific thermocline anomalies $H$ and eastern SSTA $T$ by the following form:

$$
\begin{aligned}
\frac{d T}{d t} & =-b(t) T+\omega_{0} H+\sigma \xi, \\
\frac{d H}{d t} & =-\omega_{0} T, \\
\frac{d \xi}{d t} & =-r \xi+w(t) .
\end{aligned}
$$

In this model, $-b$ is the ENSO growth rate of SST anomalies (Stein et al. 2010). The relationship between the growth rate in recharge oscillator model and AR(1) model can be further checked in appendix D. Note that $b(t)$ is seasonally varying, which is the cause of ENSO phase locking of variance (Chen and Jin 2020) and SPB (Levine and McPhaden 2015). The term $\omega_{0}$ is ENSO linear frequency (control ENSO cycle). In this paper, we set $\omega_{0}=\pi / 24$ month $^{-1}$ (Stein et al. 2010). Also, $\sigma$ is the noise amplitude, $w(t)$ is white noise, and $\xi$ is red noise with a decay time scale of $1 / r$.

\section{Seasonal persistence barrier forced by growth rate}

\section{a. Seasonal persistence barrier in relatively strongly damped system}

We first discuss a case of strong annual mean damping $(\bar{a}=0.7)$ in this subsection. This case is relatively easier to examine the relationship between the modulation of growth rate and the SPB, because the lag of the response is not large according to Liu et al. (2019).

To illustrate that the SPB is not caused by the minimum growth rate, we discuss two cases (Fig. 1a). In particular, the sharp change in the seasonal cycle of the growth rate (Fig. 1a) is designed to examine the relationship between the timing of SPB and growth rate. For profile $b$, the growth rate reaches its minimum in February, then increases sharply to its maximum in March and remains constant to September, when it declines sharply in October, and then lasts to January before its decrease to the minimum in February. Using this seasonal growth rate in Eqs. (2.2) and (2.3), the variance and persistence map are shown in Fig. 1b. While the minimum growth rate is in February, the minimum variance occurs in March, lagging the growth rate by one month. As discussed previously (Liu et al. 2019; Jin et al. 2019a), the phase difference between the seasonal growth rate and seasonal variance depends on the annual mean growth rate $\left(\bar{a}=1 / 12 \sum_{m=1}^{m=12} a_{m}\right)$. Profile $\mathrm{b}$ has a lag of 1 month because it has a rather strong damping $(\bar{a}=0.7)$. Now, the variance decreases from October to March, with the maximum reduction occurs in October (left panel of Fig. 1b, red plus sign), 1 month lagging the growth rate. The SPB, however, occurs in November (right panel of Fig. 1b). This case shows that the SPB seems to follow the months of maximum growth rate decline (GRD) time (September) or maximum variance decline (October), rather than the minimum growth rate (February). In this section, as $\sigma_{N m}^{2}=1$, according to Eq. (2.4), $\mathrm{SNR}_{m}$ is proportional to the seasonally varying variance, such that the decreasing variance corresponds to the decreasing SNR. Therefore, the SPB also follows the months of maximum SNR decline.

To confirm the role of GRD in determining the SPB, we study the second case, in which the minimum growth rate still remains in February (the same as profile b), but the GRD occurs earlier, now in June (profile c in Fig. 1a). Here, for a fair comparison, we have also kept the magnitude of the annual mean $\bar{a}$ and GRD $(\delta a)$ the same as in profile b, since both parameters are important in determining the timing of SPB (Liu et al. 2019), a point to be returned later. Now, the minimum variance still occurs in March as in Fig. 1b, whereas the maximum variance decline occurs earlier, following the GRD in July (left panel of Fig. 1c). Meanwhile, the timing of SPB is also shifted earlier to September (right panel of Fig. 1c), following the shift of the GRD. The comparison of Figs. 1b and 1c suggests clearly that the timing of SPB (November in Fig. 1b, and September in Fig. 1c) is determined by the maximum GRD (October in Fig. 1b, July in Fig. 1c), rather than the timing of the minimum growth rate (February). A further discussion of the minimum growth rate can be checked in appendix B.

To examine systematically the factors that determines the $\mathrm{SPB}$, especially the minimum growth rate (variance) or the maximum GRD, we perform a set of experiments by varying the timing of the maximum GRD from March to December while keeping the minimum growth rate fixed at February [growth rate profiles are similar to those in profiles b and $\mathrm{c}$ (Fig. 1a); $\bar{a}$ and $\delta a$ are still unchanged]. It is seen that the timing of SPB (black square) varies almost linearly with the maximum GRD (black line) [or the maximum variance decline time (red plus sign)] (Fig. 1d). In contrast, the minimum variance remains unchanged in March. This set of experiments further suggests that the SPB is generated by maximum GRD, instead of minimum growth rate. This role of GRD may seem reasonable in retrospect, because the definition of the SPB is the maximum persistence decline for the specific initial month, instead of the lowest persistence in the specific lag month. This maximum persistence decline seems to be produced by the GRD, instead of the lowest growth rate.

\section{b. Seasonal persistence barrier in weakly damped system}

We now further examine the relationship between the GRD and SPB in the case of a weak damping $(\bar{a}=0.9)$. With a weak damping, the relationship between GRD and SPB may be less obvious as the timing of SPB will be delayed by the delayed response (weak damping system), according to Liu et al. (2019). Figure $2 b$ exhibits a case of the same seasonal cycle of growth rate as the profile $b$ in Fig. 1b, with the maximum GRD in September, except for a weaker annual damping rate. The shapes of seasonal variance in both cases are similar (peaking in October in both Figs. $2 \mathrm{~b}$ and 1b). This similarity is caused by 
(a) Seasonal Growth Rate

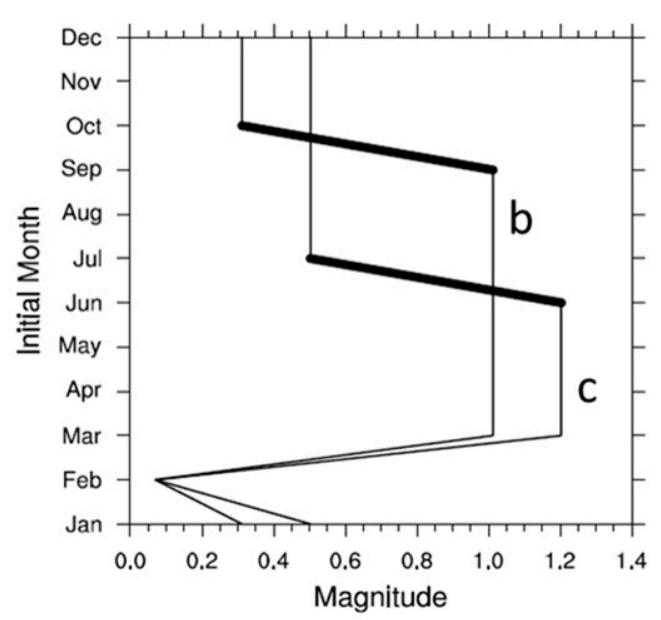

(c)

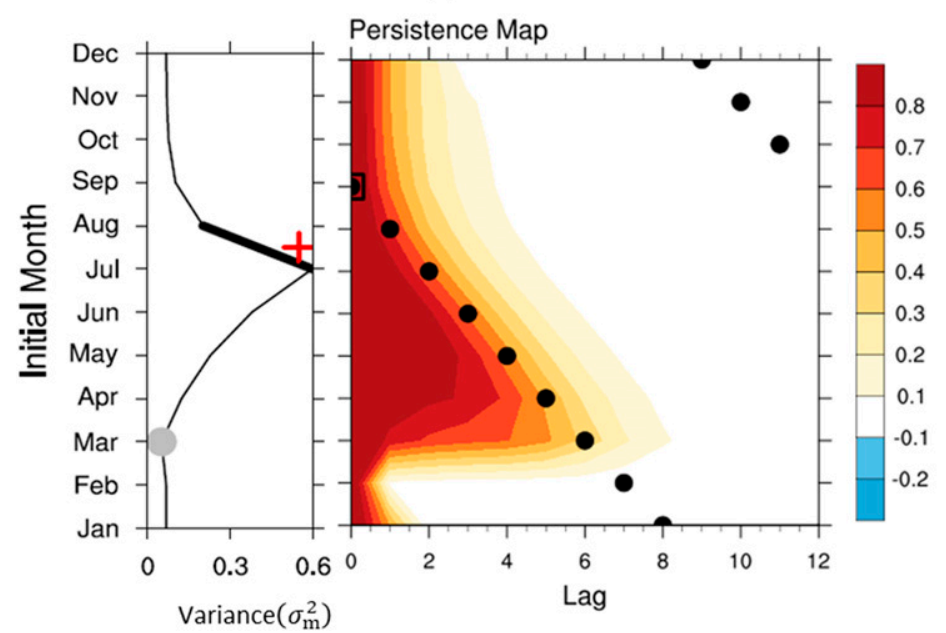

(b)

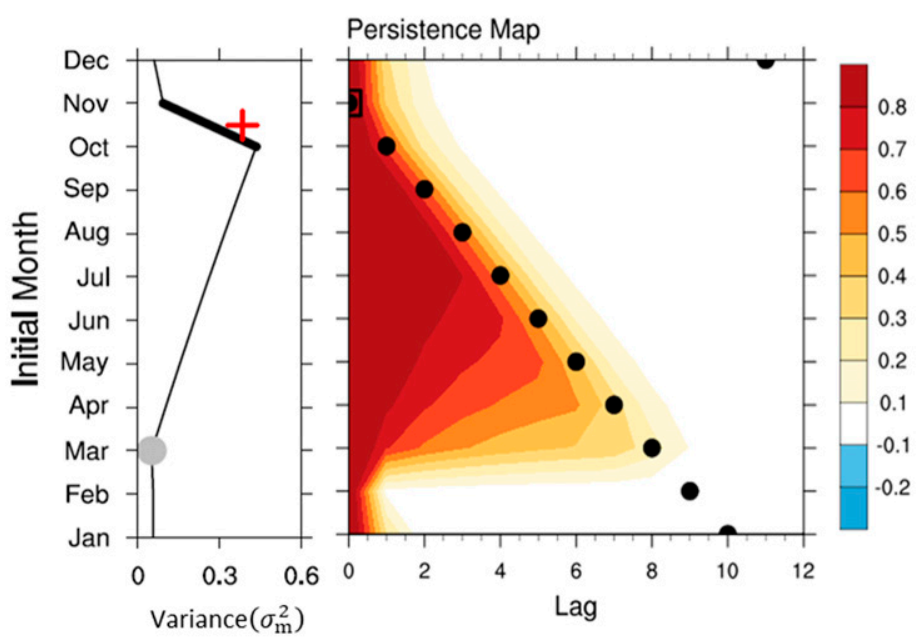

(d)

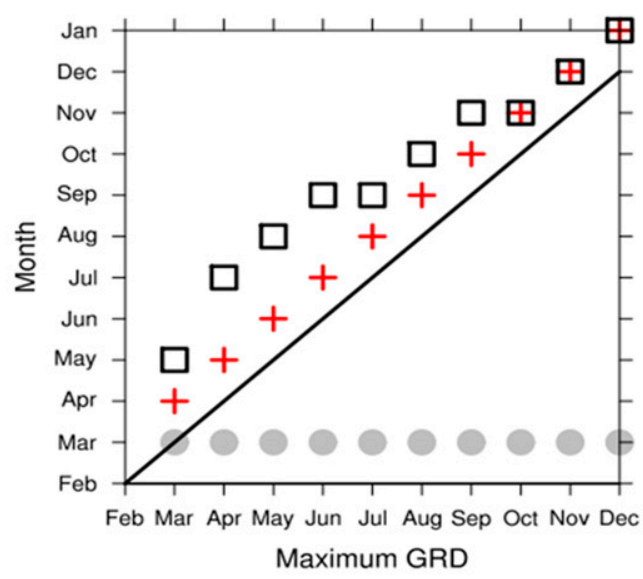

FIG. 1. The monthly growth rate, variance, and lagged correlation of a cyclo-stationary AR(1) process [Eq. (2.1)]. (a) The seasonal cycle of growth rate for profile $b$ and profile $c$, respectively. The thick line suggests the timing of the maximum growth rate decline (GRD). The variance and persistence map are forced by (b) profile b and (c) profile c. For each panel, seasonal variance is shown at left (the gray circle means minimum variance, the red plus sign shows the maximum variance declines, and the thick line indicates the variance declines caused by the maximum growth rate decline) and the persistence map is shown at right (the black circles on the persistence map mark the month of maximum persistence decline, and the black square suggests the timing of SPB). (d) The minimum variance (gray circle), maximum variance declines (red plus sign), and timing of SPB (black square) are forced by the different seasonal cycle of growth rates like in (a) with their maximum growth rate decline ranges from March to December. The black line means the SPB month is equal to the timing of the maximum GRD.

the nonharmonic annual cycle. The SPB timing is, however, delayed to February in the weak damping $\bar{a}=0.9$ case. This delay makes the SPB timing appearing to be consistent with that of minimum growth rate, which may give one the wrong impression that the SPB is caused by the minimum growth rate. If the maximum GRD is shifted early to June (profile $\mathrm{c}$ in Fig. $2 \mathrm{a} ; \bar{a}$ and $\delta a$ are the same as profile b in Fig. $2 \mathrm{a}$ ), the SPB is also shifted earlier to December (Fig. 2c). Now the timing of SPB seems to lag the maximum GRD by 6 months, in contrast to in the strong damping cases (Figs. 1b,c), when the lag is about 3 months. This shift of SPB is reasonable given the weak damping and in turn delayed response of the system.

This delay between SPB and maximum GRD compared with strong damping cases is seen more systematically in a set of experiments with the timing of maximum GRD changed systematically, while fixing the minimum growth rate at February (Fig. 2d, similar to Fig. 1d). Overall, the timing of GRD is still correlated positively with that of SPB. However, the timing of SPB lags that of maximum GRD by about 3-6 months (Fig. 2d), which is systematically longer than the 
(a) Seasonal Growth Rate

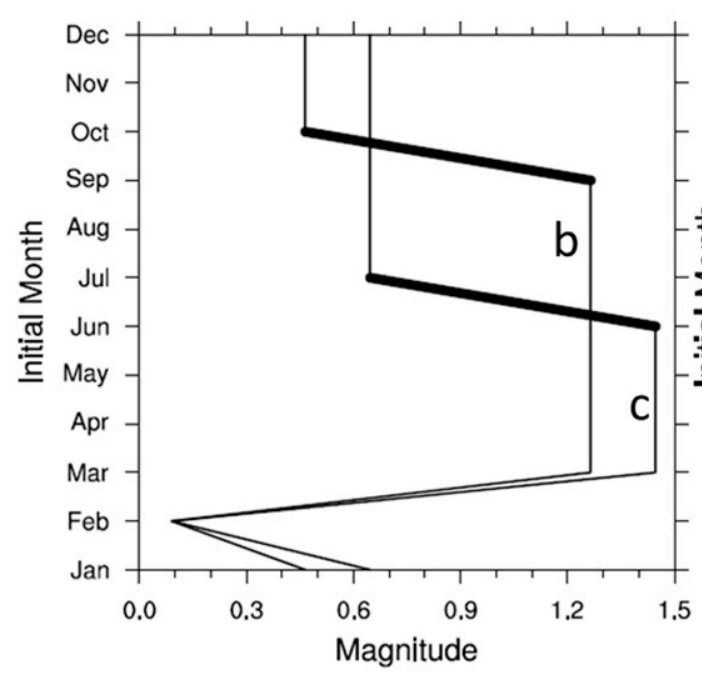

(c) (b)

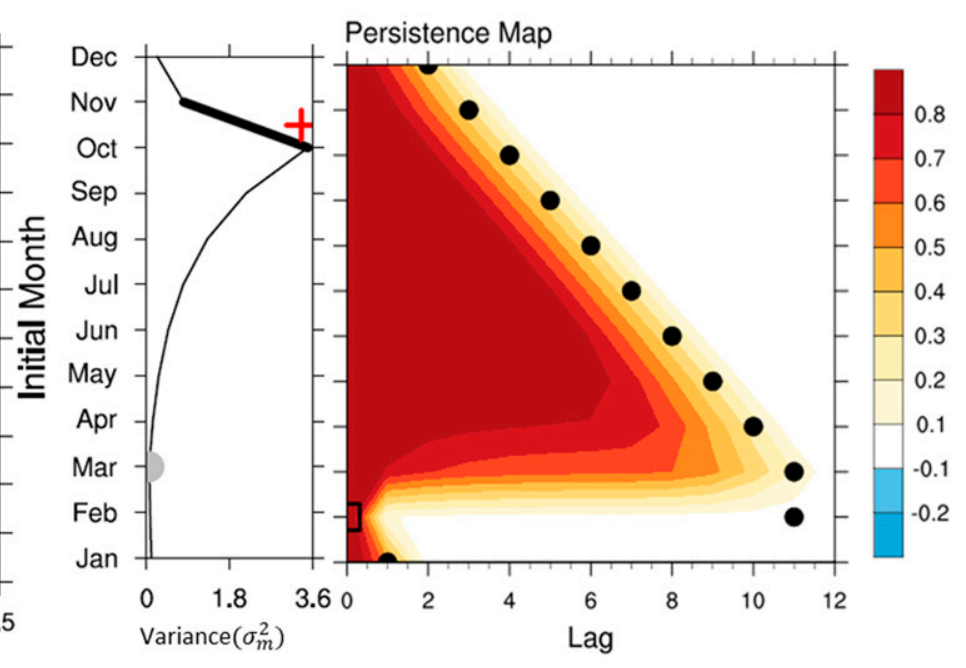

(d)
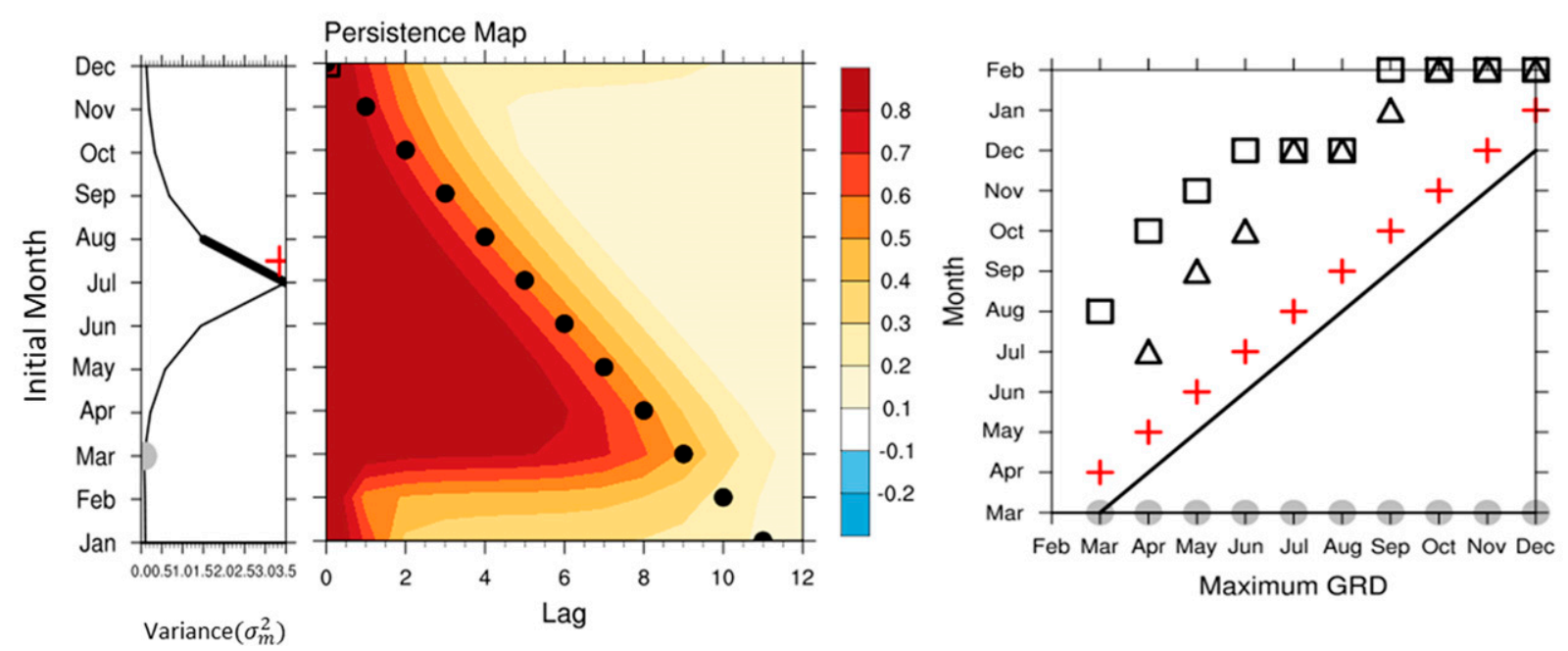

FIG. 2. As in Fig. 1, but for $\bar{a}=0.9, \delta \mathrm{a}=0.9$ cases. Here in (d), black triangles mean the PB forced by the cases when $\bar{a}=0.9, \delta a=0.2$.

1-4 months in the strong damping cases (black squares in Fig. 2d vs Fig. 1d), while the minimum variance remains unchanged at March. This delayed response of SPB is consistent with the weaker damping and in turn longer response time. Therefore, the weak damping may make the SPB appearing lagging from maximum GRD.

One feature different between the weak damping cases (Fig. 2d) and strong damping cases (Fig. 1d) is that, when the maximum GRD ranges from October to December, the SPB remains locked in February. Therefore, for the cases of weak damping (large $\bar{a}$ cases, more relevant to observational ENSO), the SPB problems seem to be more complicated, because it may involve the interference of growth rate increase on growth rate decrease, as discussed in appendix $\mathrm{C}$.

In sum, the SPB is generated mainly by the maximum GRD, rather than the minimum growth rate. Furthermore, the SPB will be delayed from the GRD in the case of weak damping. This also helps us to explain why SPB occurs during the period of increasing growth rate in the case of weak damping both in the theoretical solution of Liu et al. (2019) and in the observational ENSO, a point to be returned later in section 5 .

\section{Seasonal persistence barrier forced by noise forcing}

In this section, we will discuss the role of noise forcing on SPB. As suggested by Liu et al. (2019), a seasonal cycle of noise forcing will also cause the SPB. As such, we set the growth rate as a constant $a_{m}=\bar{a}$ and the noise forcing with a nonharmonic annual cycle.

Similar to the case of seasonal growth rate, we will show that it is the increasing noise forcing (INF), rather than the maximum noise forcing as one is tempted to assume, that leads to 
(a) Seasonal Noise Forcing

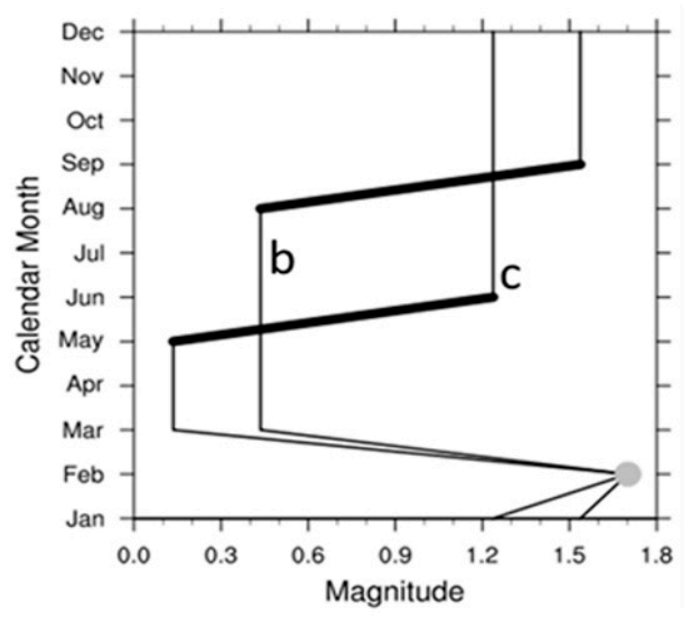

(c) (b)

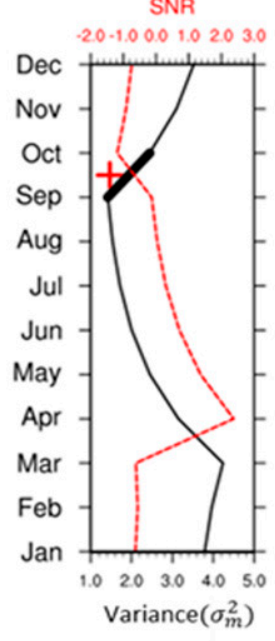

Persistence Map

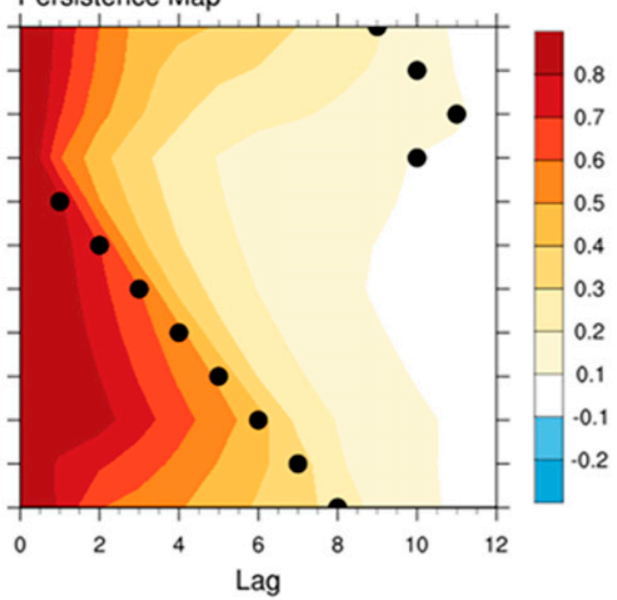

(d)
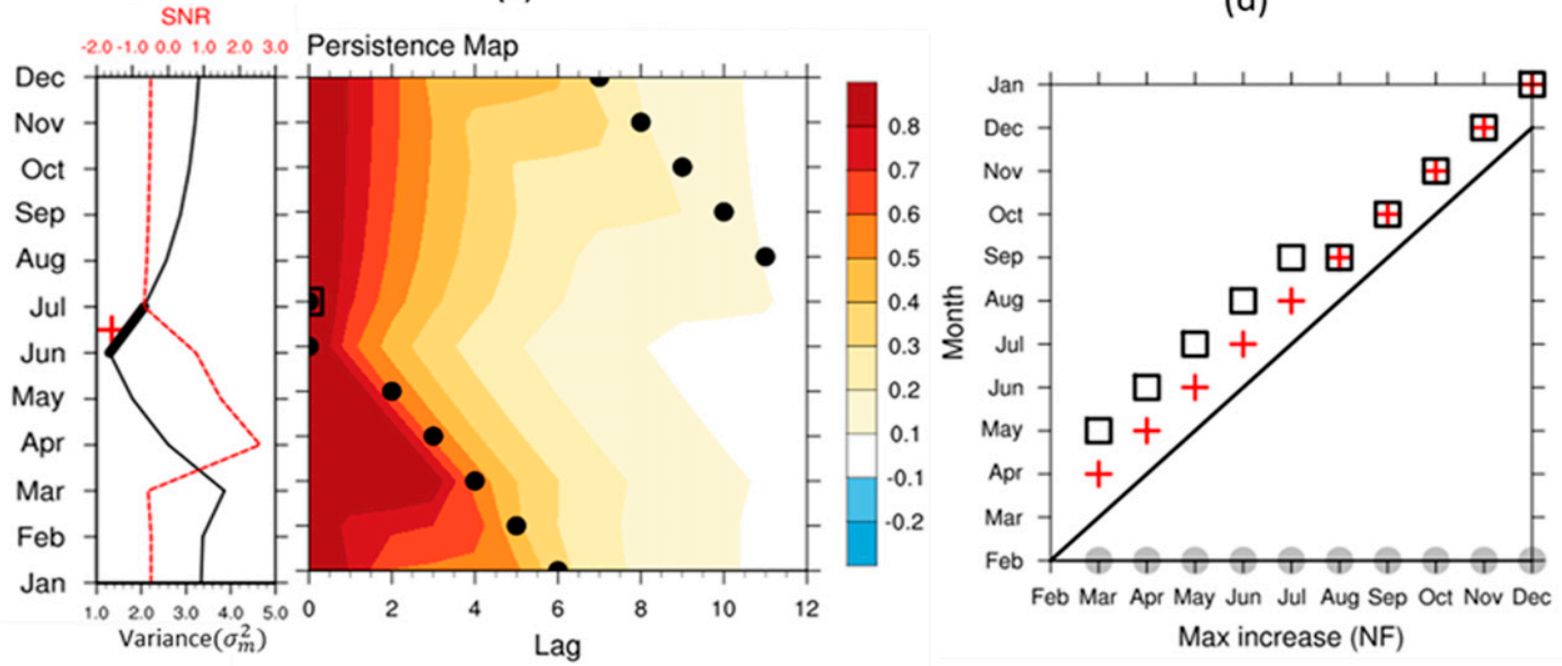

FIG. 3. The monthly noise forcing, variance, and lagged correlation of a cyclo-stationary AR1 process [Eq. (2.1)]. As in Fig. 1, but for the noise forcing case $(\bar{a}=0.8)$. The red line in (b) and (c) is the signal-to-noise ratio line. The gray circle in (a) and (d) is maximum noise forcing month. The red plus sign in (d) means the maximum variance increases.

the SPB. For profile b in Fig. 3a, the noise forcing reaches its maximum in February, then decreases sharply to its minimum in March and remains constant in August, when it increases sharply in September, and then lasts to January before it increases to its maximum in February. Using this seasonal cycle of noise forcing (profile $\mathrm{b}$ in Fig. 3a) and growth rate $(a=0.8)$ in Eqs. (2.2) and (2.3), we obtain the variance (left panel of Fig. 3b) and persistence map (right panel of Fig. 3b) forced by the noise forcing. It shows that the SPB occurs in September, which is in the period of increasing noise forcing (September), instead of the maximum noise forcing (February). Meanwhile, the variance is also increasing during this period. This is opposed to the growth rate case that the variance is decreasing at the timing of SPB.

This concurrence of the SPB with increasing variance can be explained in terms of SNR in Eq. (2.4). For the growth rate case, as the noise forcing is constant for all the year around $\sigma_{N m}^{2}=1, \mathrm{SNR}_{m}=\left(\sigma_{m}^{2}-\sigma_{N m}^{2}\right) / \sigma_{N m}^{2}$ is proportional to the varying variance $\sigma_{m}^{2}$. However, for seasonal noise forcing, the noise forcing plays dual roles of forcing signal $\left(\sigma_{m}^{2}-\sigma_{N m}^{2}\right)$ and noise $\left(\sigma_{N m}^{2}\right)$. The increase of variance signal is also caused by the increase of noise forcing. Therefore, the ratio, or SNR, no longer follows the variance. In the case of Fig. 3b, when the variance signal $\sigma_{m}^{2}$ in the numerator increases, the SNR decreases (red line in the left panel of Fig. 3b) because of the increase of noise $\sigma_{N m}^{2}$ in the denominator; and this decrease of SNR causes the SPB. These features are also found in the example in Fig. 3c, whose variance and persistence map are forced by profile $\mathrm{c}$ in Fig. 3a. In this case, the timing of maximum INF is moved to June while still keeping the maximum noise forcing at February. The SPB is also shifted to after INF in July, when the variance increases and the SNR decreases. 


\section{(a) Seasonal Noise Forcing}

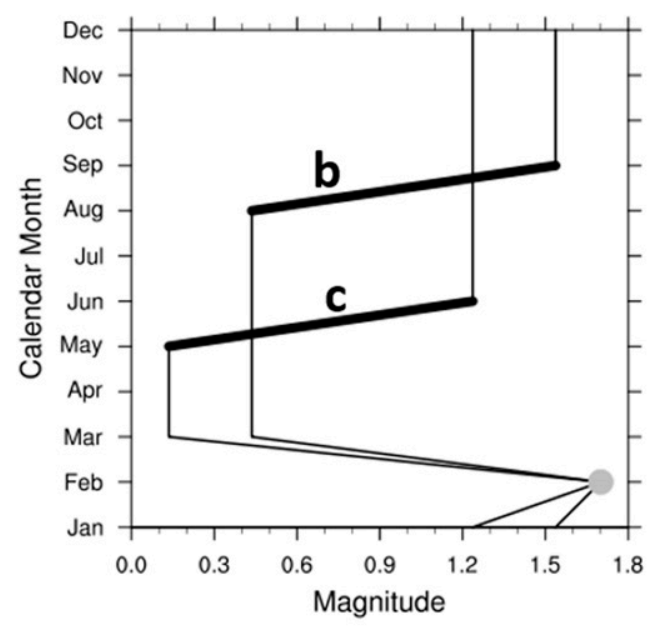

(c)

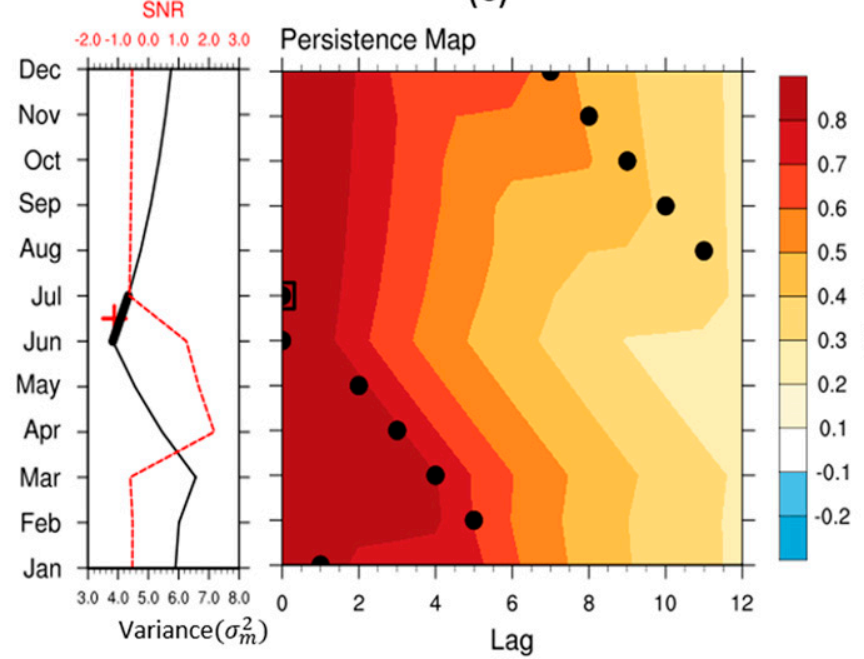

SNR

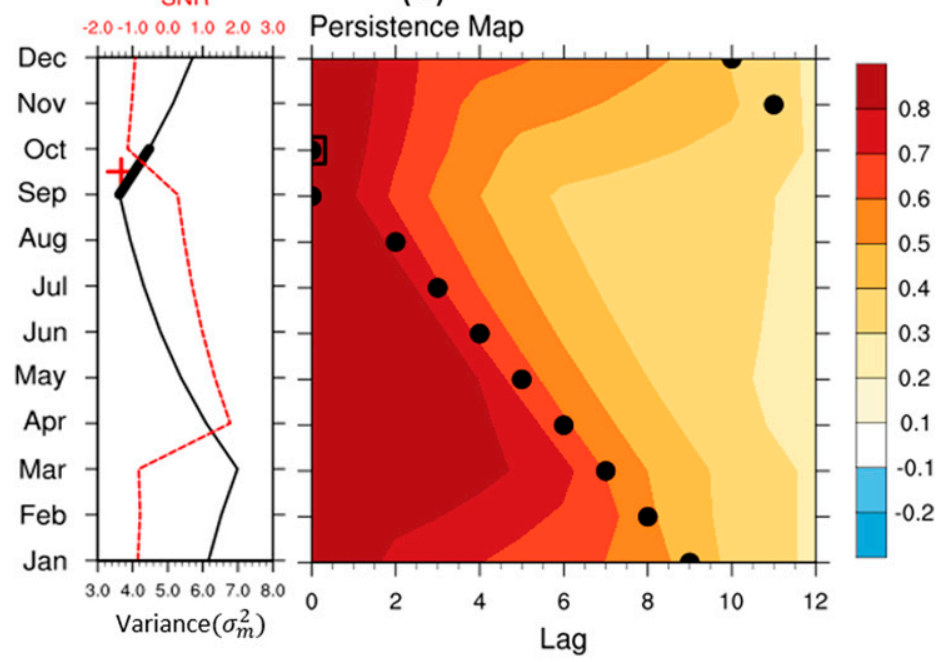

(d)

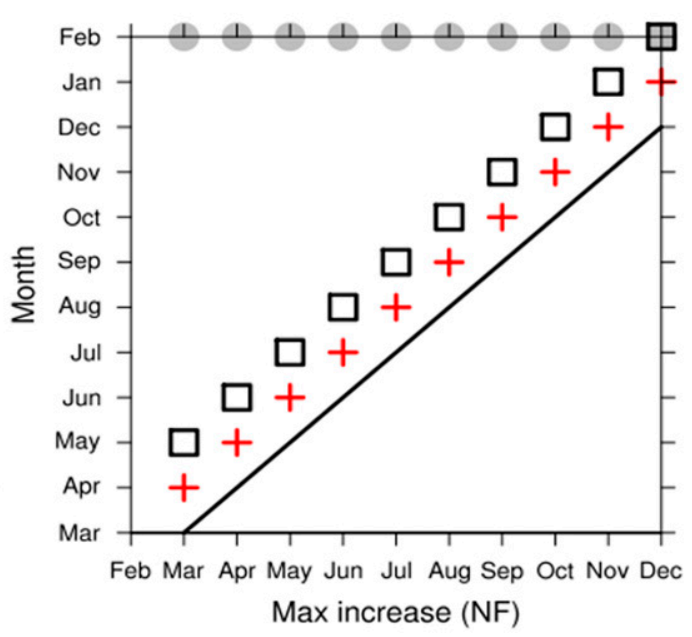

FIG. 4. As in Fig. 3, but for the $\bar{a}=0.9$ case.

To further confirm the role of increasing noise forcing with regard to the SPB, we keep the maximum noise forcing in February, but shift the timing of the maximum INF from March to December systematically. It is seen that the SPB months and the timing of maximum variance are both shifted, while the maximum noise forcing remains at February (Fig. 3d), further demonstrating that the latter is not essential for the SPB.

The relationship between the SPB month and the timing of maximum INF can also be seen for a weaker damping $\bar{a}=0.9$ in Fig. 4. Here, we keep the shape of the noise amplitude (profiles $b$ and $\mathrm{c}$ in Fig. 4a) the same as Fig. 3a, except that $\bar{a}$ is increased to $\bar{a}=0.9$. Again, the SPB timing increases with that of the INF, similar to the case of $\bar{a}=0.8$ (Fig. $4 \mathrm{~d}$ vs Fig. $3 \mathrm{~d}$ ). In comparison with the case of seasonal growth rate forcing, the annual mean damping rate plays a less important role in the SPB month here. This reduced sensitivity of SPB to mean damping rate may be due to two reasons. First, in the growth rate case, when $\bar{a}$ increases to a larger value, in some months $a_{m}$ may exceed 1 when the system becomes unstable and will grow by itself. This strong intensification of the signal may overwhelm the signal in the damped period driven by the noise forcing, complicating the signal and in turn SPB. However, for the case of noise forcing, the system is damped all the time $\left(a_{m}<1\right)$, which is relatively straightforward to understand. Second, according to Eq. (2.3), the SPB is caused both by the variance ratio (variance is also controlled by $a_{m}$ ) and by $a_{m}$ in the growth rate case. However, for the noise forcing case, the SPB is only caused by the variance ratio ( $a_{m}$ is constant all months around). Therefore, the role of the growth rate is much harder to understand compared with noise forcing case, especially for weak damping cases when $\bar{a}$ is close to 1 .

In short, for the noise forcing case, the SPB month, as well as the timing of maximum variance increase, is caused by the maximum INF, because it leads to maximum decrease of SNR. The maximum noise forcing (or weakest SNR) is related more 
(a) Tropical Pacific

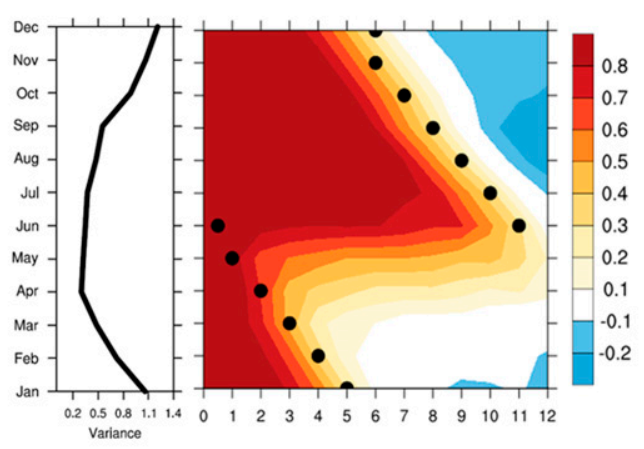

(c) Seasonal Cycle GR (only change the slope)

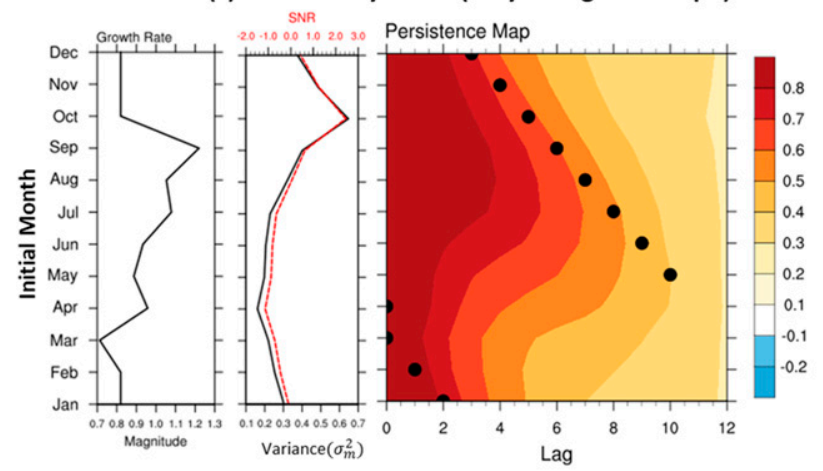

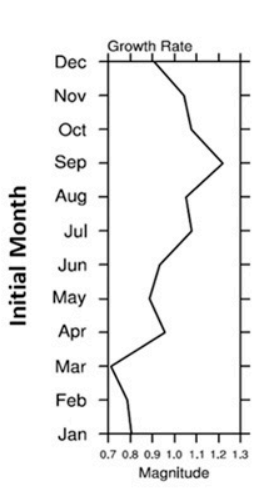

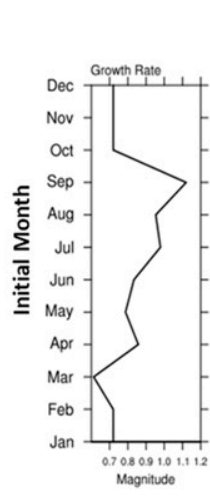

(b) AR(1) model

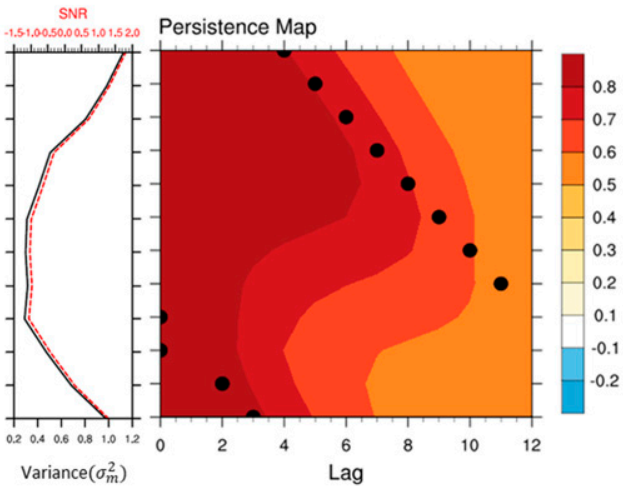

(d) Small annual mean GR

SNR $20-1000$ 10 2030 Persistence Map
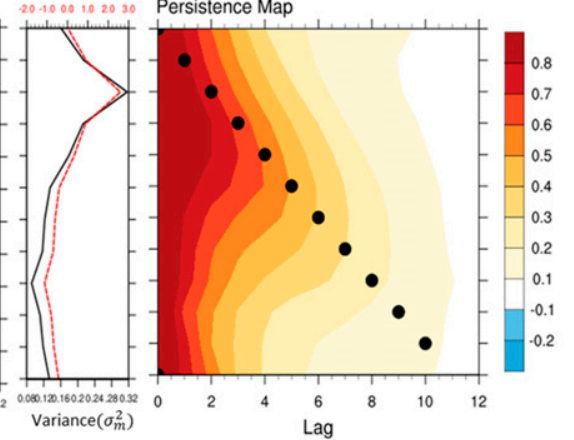

FIG. 5. (a) The seasonal variance and autocorrelation function (persistence map) in the tropical Pacific (Niño-3.4 region; $5^{\circ} \mathrm{S}-5^{\circ} \mathrm{N}, 170^{\circ}-$ $120^{\circ} \mathrm{W}$ ) calculated directly from monthly SST anomaly in the observation. (b) The seasonal cycle of growth rate calculated by tropical Pacific SST is shown in the left panel. Seasonal variance (black) and SNR (red) and persistence map, shown in the center and right panels, respectively, are forced by this seasonal growth rate. (c),(d) As in (b), but for different seasonal growth rate cases.

to the lag-1 autocorrelation coefficient, instead of the SPB, as discussed by Jin et al. (2019a).

\section{Application to the tropical Pacific and North Pacific}

In sections 3 and 4, we show that either a decreasing growth rate or an increasing noise forcing will lead to a SNR decrease, and in turn the SPB. Here, we will apply this understanding to the tropical Pacific and North Pacific SSTA. We will use the monthly sea surface temperature data from the Hadley Centre Sea Ice and Sea Surface Temperature (HadISST) from 1960 to 2005 (Rayner et al. 2003). The SSTA is derived after subtracting the climatological seasonal cycle and linear trend. The monthly growth rate and noise variance are obtained from the observation using the AR(1) model solution (Liu et al. 2019).

We first discuss the case of tropical Pacific SSTA variability of ENSO, where the SSTA minimum variance occurs in April and the SPB occurs in May-June, as shown in the persistence map of the observed SST (Fig. 5a, also see Liu et al. 2019). This so-called spring SPB has been recognized to be forced by the seasonal growth rate (Liu et al. 2019). It can be studied here using the AR(1) model by keeping the annual cycle of growth rate the same as from the observation (left panel of Fig. 5b) but changing the noise forcing magnitude as the annual mean constant throughout the year. The variance and persistence map can be derived from Eqs. (2.2) and (2.3), respectively. The right panel of Fig. 5b shows a distinct SPB phase locked to late spring (April). This spring SPB is caused by the seasonal growth rate, which is consistent with Levine and McPhaden (2015).

In the one-dimension model of tropical Pacific, the SPB is caused by the GRD from September to March in the presence of a weak annual mean damping. We verify this by changing the slope of the GRD from September to March to be confined to a single month of September-October (left panel of Fig. 5c), while keeping the rest of growth rate (including the minimum growth rate in March) the same as the original growth rate (left panel of Fig. 5b). The forced persistence map (right panel of Fig. 5c) shows that the SPB is shifted early from April to March. This can be understood as the GRD occurs slightly earlier and therefore shifts the SPB slightly earlier. On the other hand, if we decrease the annual mean growth rate from $\bar{a}=0.91$ to $\bar{a}=0.81$ while keeping the shape of seasonal growth rate the same as the left panel of Fig. 5c, the SPB month is shifted earlier by $\sim 3$ months to December (right panel of Fig. 5d). This suggests that the weak damping rate (large annual mean growth rate) in the observed ENSO delays the SPB from immediately after the GRD (December) to 3 months later (in spring). Therefore, the well-known ENSO spring SPB 


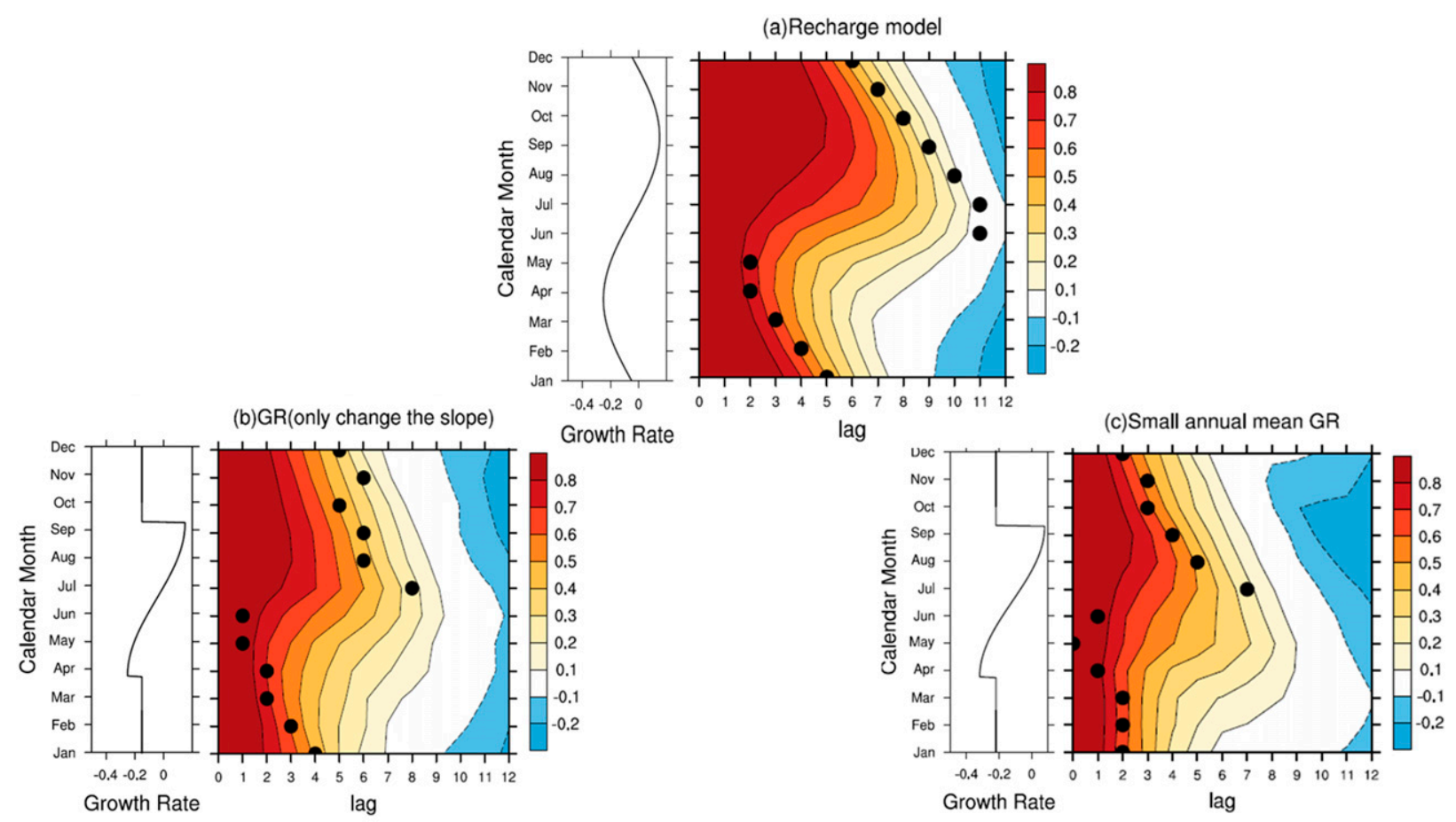

FIG. 6. As in Figs. 5b-d, but for the recharge model. Here the relationship between the growth rate $-b$ in the recharge model and the growth rate $a$ in the $\mathrm{AR}(1)$ model is $-b=a-1$ (see appendix D).

is caused by the GRD from autumn to spring and then further delayed by the weak damping rate to late spring. This spring timing of SPB occurs coincidentally with, but is not caused by, the minimum growth rate in spring.

The decreasing growth rate from September to March and weak damping rate cause ENSO spring SPB in the numerically solution of the recharge oscillator model [Eqs. (2.5)-(2.7)], which is consistent with the results using AR(1) model (Fig. 6 vs Fig. 5). Forced by an idealized seasonal growth rate on an annual harmonic (left panel of Fig. 6a), both the recharge oscillator model and the AR(1) model show that the seasonal growth rate could cause the ENSO spring SPB [Fig. 6 herein vs Fig. 5d in Liu et al. (2019)]. The main difference between two models is that the negative ACF occurs in the recharge oscillator model. We change the slope of the decreasing growth rate while keep the rest value the same with left panel of Fig. 6a. Figure $6 \mathrm{~b}$ suggests that the timing of SPB moves from June to May. Then, we decrease the annual mean growth rate while keeping the shape of seasonal growth rate the same as the left panel of Fig. 6b. The SPB month happens in March (Fig. 6c), which is two months earlier than the large growth rate case (Fig. 6b). The results are similar to those in the AR(1) model, which show that these two models have the similar sensitivity for SPB.

As the damping rate (or annual mean growth rate $\bar{a}$ ) plays an important role in the timing of ENSO SPB, we want to emphasize the role of $\bar{a}$ in physics. Physically, it describes the strength of large-scale ocean-atmosphere feedback. For the tropical Pacific, it can be assessed in terms of the Bjerknes stability index (Jin et al. 2006, 2019b). An increase (decrease) in $\bar{a}$ means that the system becomes more (less) persistent. As such, it can be used to identify the decadal modulation of the ENSO variability after the twenty-first century (e.g., Lübbecke and McPhaden 2014)

Seasonal noise forcing has been recognized as the main cause for the SPB in North Pacific (Liu et al. 2019). The SSTA variability in the North Pacific can also be simulated in an AR(1) model. Note, the noise forcing in our model Eq. (2.1) indicates the "effective noise forcing," which is proportional to the atmospheric noise forcing divided by the surface ocean heat content. Physically, an increasing noise forcing in the North Pacific is caused by a shallowing mixed layer in the summer. Figure 7a suggests that variance peaks in the August and SPB occurs in June for North Pacific (Zhao et al. 2012; Liu et al. 2019). The role of seasonal noise forcing on the SPB here can be seen in an AR(1) model in Fig. 7b. The annual cycle of noise forcing remains the same [left panel of Fig. 7b; same as Liu et al. (2019)] while the growth rate is replaced with the annual mean growth rate. Taking this seasonal noise forcing and growth rate to Eqs. (2.2) and (2.3), an SPB still remains largely unchanged, with the month shifted slightly earlier to May (Fig. 7b) when the variance (SNR) increases (decreases).

In contrast to ENSO, the INF from March to June is now the key factor that determines the SPB in North Pacific. The large noise forcing, however, is related to the small lag-1 autocorrelation (Jin et al. 2019a), not the SPB. As discussed in section 4, the INF is the cause of SPB. To see more clearly the formation mechanism for the SPB in the North Pacific, we change the slope of the INF from March to June to be confined to a single month of February to March while keeping the 
(a) North Pacific

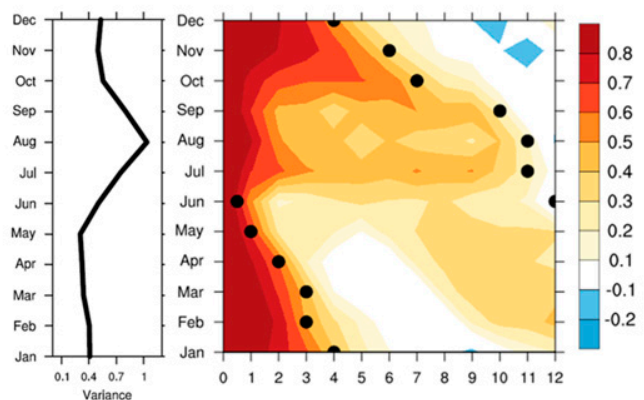

(c) Seasonal Cycle NF (only change the slope)

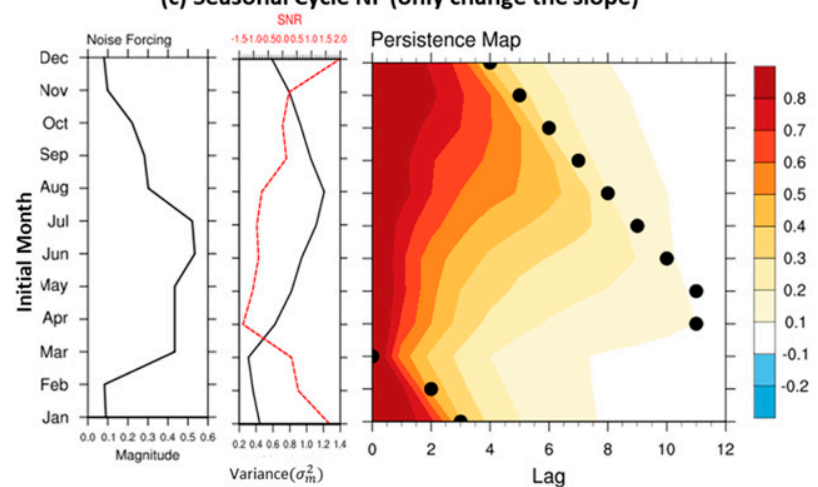

(b) AR(1) model
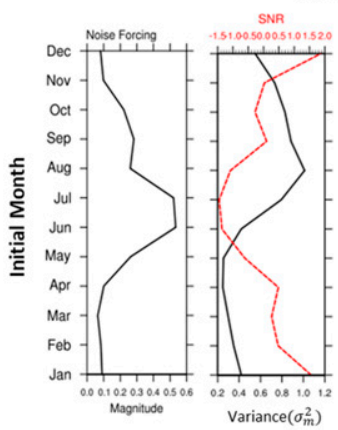

Persistence Map

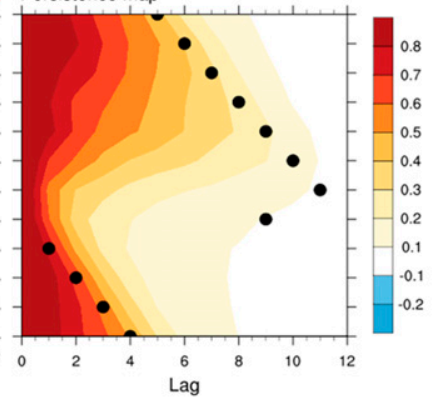

(d) Small annual mean GR

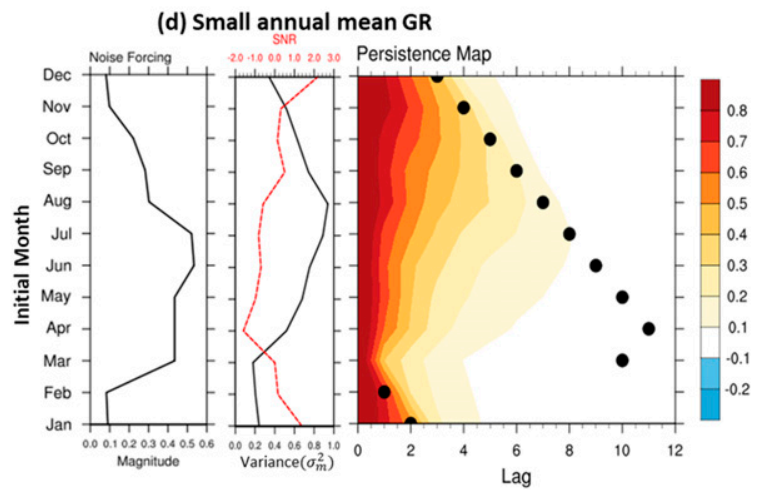

FIG. 7. As in Fig. 5, but for North Pacific $\left(38^{\circ}-42^{\circ} \mathrm{N}, 160^{\circ} \mathrm{E}-180^{\circ}\right)$ case. Here (b)-(d) are forced by the seasonal noise forcing.

maximum noise forcing in summer (left panel of Fig. 7c). The result suggests that the timing of SPB is shifted earlier from May to April (right panel of Fig. 7c). We also experiment by keeping the shape of this seasonal noise forcing, but decreasing the annual mean growth rate from 0.78 to 0.68 (stronger damping in Fig. $7 d$ ). The timing of SPB is shifted slightly earlier from April to March. Therefore, for the North Pacific, the increasing noise forcing from March to June is the main reason for the summer SPB.

\section{Summary and discussion}

As a follow up of Liu et al. (2019), this paper attempts to understand the formation mechanism of SPB in the framework of the AR(1) model. In particular, we use idealized nonharmonic seasonal forcing to help isolate the mechanism. It is found that the minimum variance is mainly modulated by the small growth rate or noise forcing. However, the SPB is caused by the maximum growth rate decreasing (GRD) or increasing noise forcing (INF), corresponding to the period of decreasing SNR, instead of the minimum growth rate or maximum noise forcing (weakest SNR). Meanwhile, a small damping rate will delay the SPB significantly up to a season. This mechanism offers an explanation that in the weak damping case [such as that in Fig. 3b of Liu et al. (2019)], the SPB occurs in the period of increasing growth rate. It also provides an explanation for the observational ENSO SPB as forced by the decreased growth rate from September to March and then further delayed by the weak damping to late spring, but not forced by the minimum growth rate in spring. In this view, we have to consider the decreasing air-sea coupled instability (growth rate) from autumn to spring as the key process of for ENSO spring SPB, rather than the low coupled instability in the spring. In an accompanying paper, we linked the mathematical growth rate with physical BJ coupled-stability index (Jin et al. 2006) to study the physical process of driving the ENSO spring SPB (Jin et al. 2019b). We also apply our mechanism to the North Pacific SSTA and find that the increasing noise forcing from March to May is the key factor that determines the summer SPB there, rather than maximum noise forcing in summer.

Given that the SPB can be caused by either decreasing growth rate or increasing noise forcing, it is possible that two SPBs can occur simultaneously, if there are two GRDs or INFs in the seasonal cycle. Figure 8 shows one idealized example in the AR(1) model for two barriers. In Fig. 8a, there are two GRDs, one in June and the other in January (left panel in Fig. 8a). The persistence map (Fig. 8a) exhibits one strong SPB in September and a very weak SPB in February. The strong SPB is caused by the GRD in June with no subsequent increase of growth rate, whereas the weak SPB is caused by the GRD in January but canceled partly by the subsequent strong increase of the growth rate in March. As the GRD intensifies in January and weakens in June (Fig. 8b), the weaker SPB is intensified while the stronger SPB is weakened, leading to comparable SPBs. If the GRD further increases in January and decreases in June, February SPB becomes the dominant SPB, while the August SPB diminishes (Fig. 8c). This phenomenon of multiple SPBs also exists in the observation. We can see it in the eastern North Pacific in the so-called reemergence region $\left(26^{\circ}-42^{\circ}, 130^{\circ}-116^{\circ} \mathrm{W}\right.$; Fig. 9). There are two very strong barriers in July and November, respectively. Therefore, our mechanism can also explain the features of more than one barrier in the real-world ocean. 
(a)

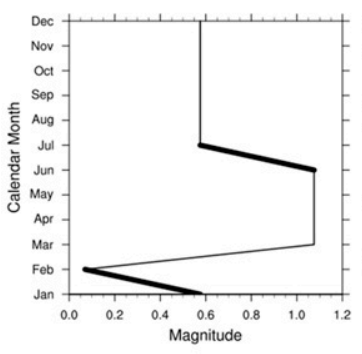

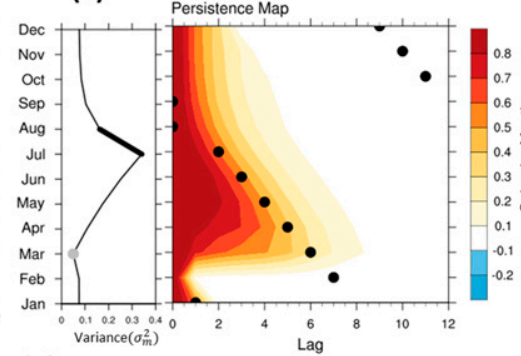

(c)

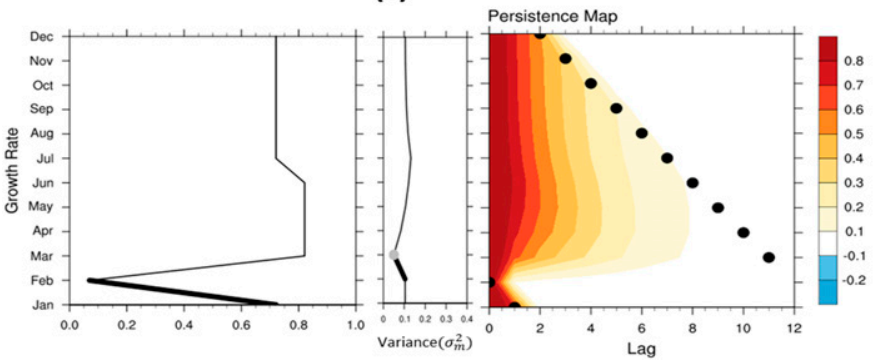

(b)

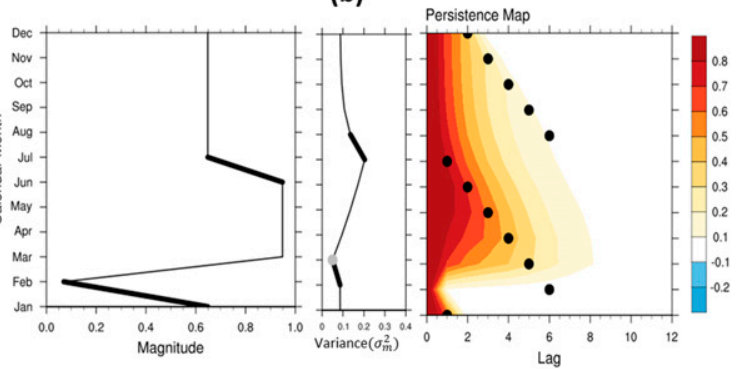

FIG. 8. (a) The variance (shown in thecenter panel) and persistence map (shown in the right panel) are forced by the seasonal growth rate (shown at left). (b),(c) As in (a), but forced by different seasonal growth rates (see their left panels).

The mechanism in this paper helps us further understand the cause of SPB and understand the SPB features in the realworld ocean. Moreover, it can serve as a null hypothesis for the SPB of climate variability in general.

Acknowledgments. We thank four anonymous reviewers for their valuable comments. This work is supported by the National Key R\&D Program of China (2017YFC1404100 and 2017YFC1404104), Chinese MOST 2017YFA0603801, NSFC41630527, and U.S. NSF AGS-1656907.

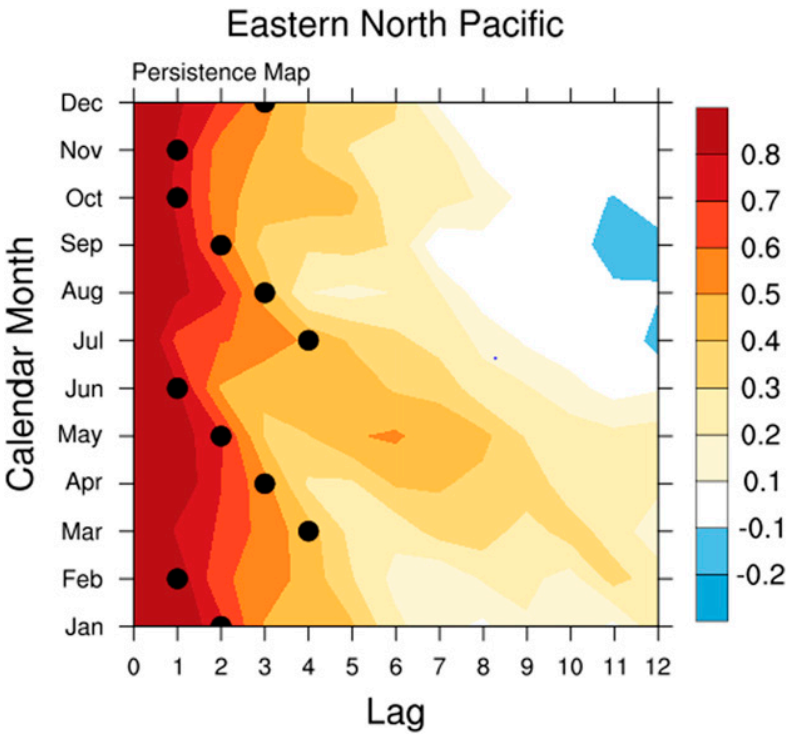

FIG. 9. As in Fig. 7a, but for the eastern North Pacific $\left(26^{\circ}-42^{\circ} \mathrm{N}\right.$, $\left.132^{\circ}-116^{\circ} \mathrm{W}\right)$.

\section{APPENDIX A}

\section{The Role of Different Kinds of Noise Forcing in SPB}

According to Levine and McPhaden (2015), in the tropical Pacific the noise is red noise, which fits better in the observation. Here in order to identify the role of different kinds of noise forcing in ENSO SPB, we use white noise forcing and red noise forcing under the seasonally varying growth rate, respectively, both in the recharge oscillator model and the $\mathrm{AR}(1)$ model. The recharge oscillator model is used in Eqs. (2.5)-(2.7). The seasonal growth rate [ $-b(t)$, the same as in Stein et al. (2010), their Fig. 0.2] is in the left panel of Fig. A1. For white noise forcing and red noise forcing, the decorrelation time scale is 0 and 45 days, respectively, which is consistent with Levine and McPhaden (2015).

The main features of persistence map for white noise forcing and red noise forcing are nearly the same, as suggested by Fig. A1. The SPB features in Figs. A1a and A1b are mainly caused by the seasonally varying growth rate, regardless of the different noise forcing.

As for the Langevin equation that incorporates a seasonal cycle in the growth rate

$$
\begin{aligned}
& \frac{d T}{d t}=-b(t) T+\sigma \xi, \\
& \frac{d \xi}{d t}=r \xi+w(t),
\end{aligned}
$$

mathematically, the Langevin equation is the special case of recharge model when $\omega=0$. Therefore, when $\omega$ is smaller, the feature between recharge model and Langevin equation is more similar.

There is also little difference for SPB in the Langevin equation between white noise forcing and red noise forcing (Fig. A2). The 
(a)White noise

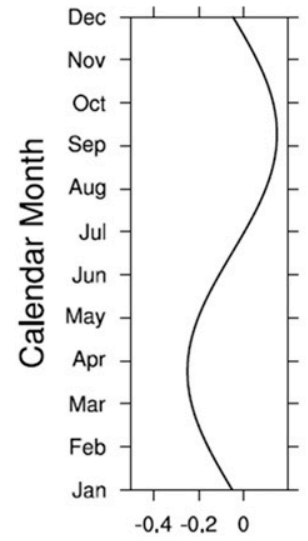

Growth Rate
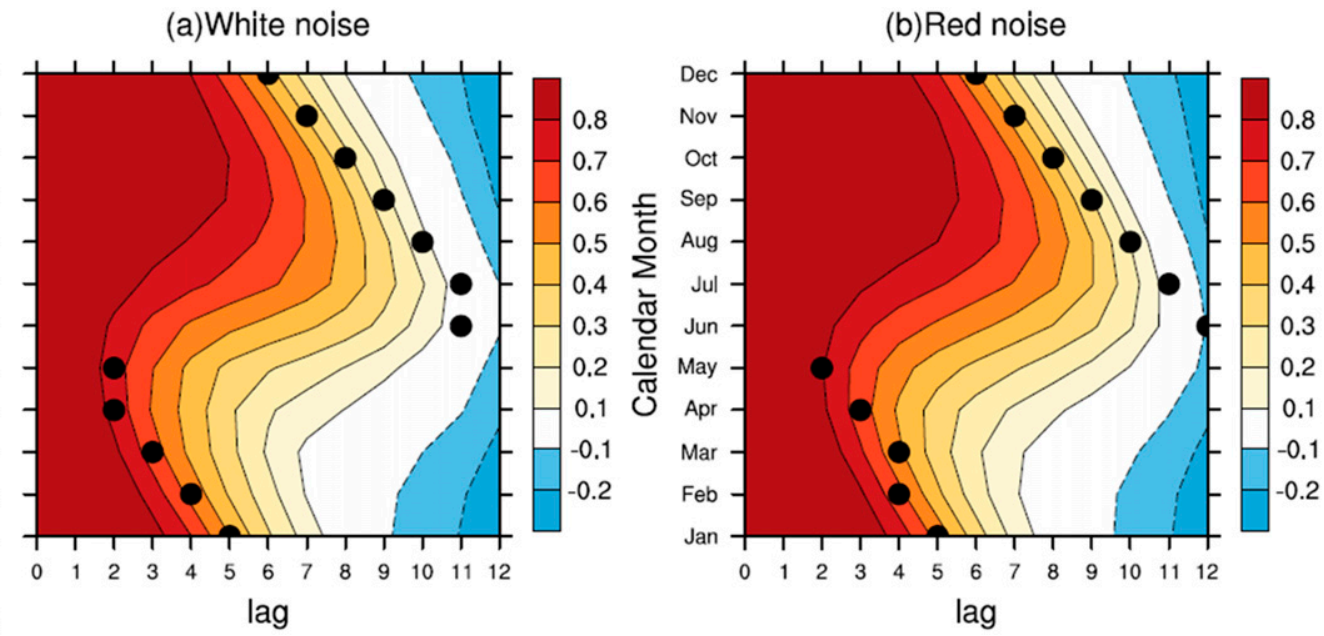

FIG. A1. The persistence map for (a) white noise forcing (shown in the right panel) and (b) red noise forcing in the recharge model. Shown in the left panel of (a) is the seasonal growth rate.

SPB features of Fig. A2 are nearly the same as in Fig. A1. It is reasonable as ENSO inherent cycle is large $\left(\omega=\pi / 24\right.$ month $\left.^{-1}\right)$. Therefore, it is not important about red noise or white noise when we consider SPB phenomenon, although red noise forcing is closer to observation. Here we use white noise, mainly because the theoretical solution of Liu et al. (2019) is based on the white noise forcing.

\section{APPENDIX B}

\section{The Role of Minimum Growth Rate}

We now confirm that the minimum growth rate leads to the minimum variance, but not the SPB, in another example in
Fig. B1. Now, the minimum growth rate is shifted from February to October, while $\bar{a}$ and the timing of GRD remain the same as in profile $\mathrm{c}$ of Fig. 1a. This shift of minimum growth rate shifts the minimum variance to November (left panel of Fig. B1), demonstrating that the minimum growth rate does determine the minimum variance. Meanwhile, shifting this minimum growth rate from February to October also produces a strong effect on the variance increase between April to the peak in July (left panel of Fig. B1). According to Eq. (2.2), the growth rate in February plays a more critical role for variance from April to July than the growth rate in October. In Fig. B1, the growth rate in February is much larger than that in the profile $\mathrm{c}$ of Fig. 1, which causes a larger forced variance in Fig. B1. Although the minimum variance is shifted because of
(a)White noise

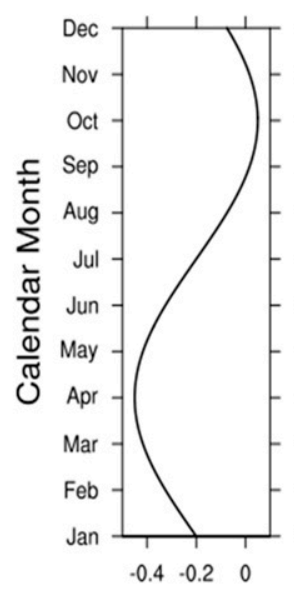

Growth Rate
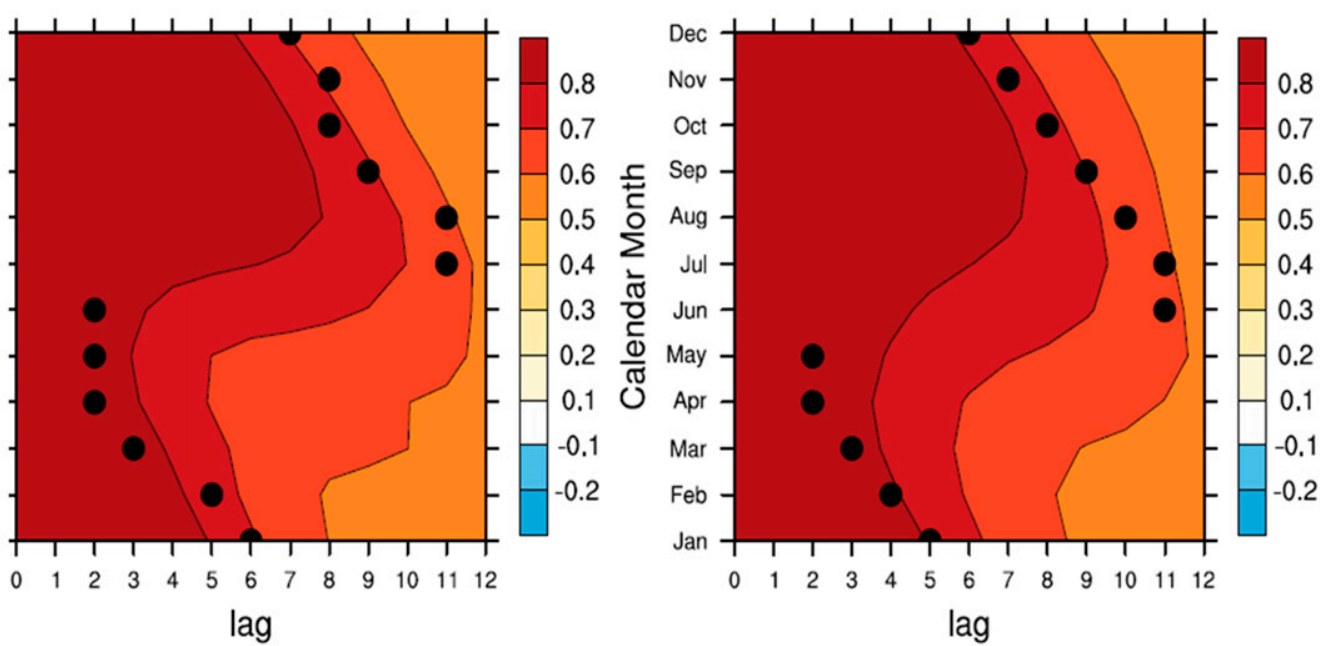

Fig. A2. As in Fig. A1, but for Langevin equation. 
(a) Seasonal Growth Rate

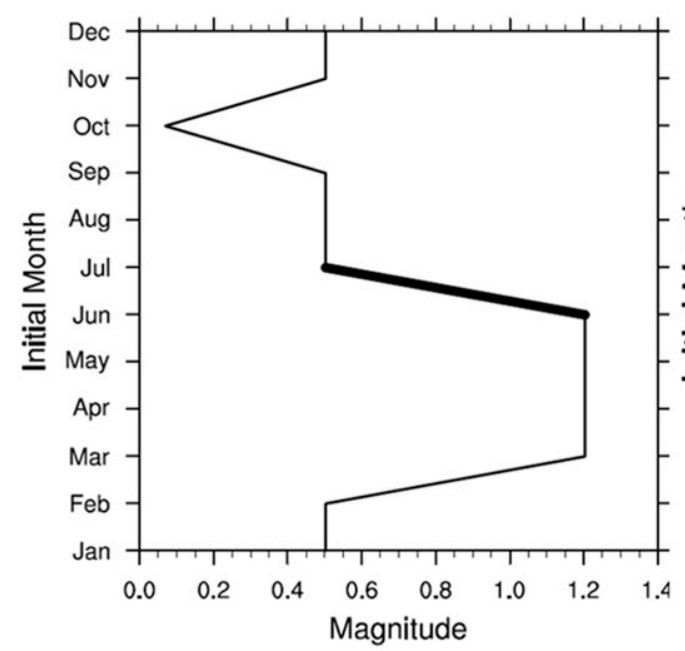

(b)

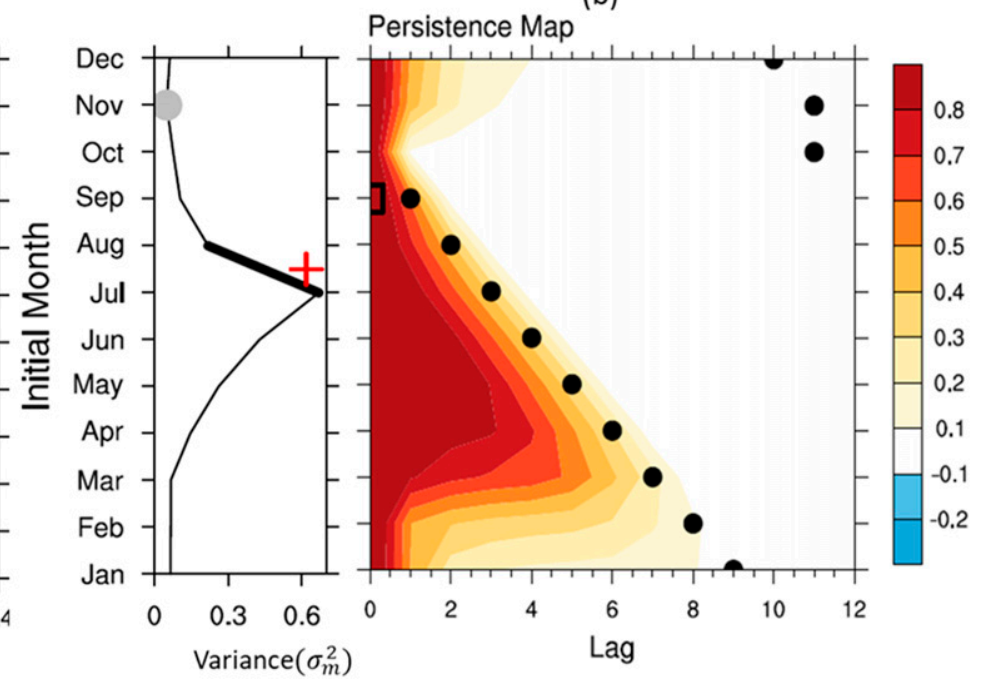

Fig. B1. As in Figs. 1a and 1c, but for different phase of growth rate case.

the minimum growth rate, the SPB month (the right panel of Fig. B1b) is almost the same as Fig. 1c. This example suggests again that the SPB is determined more by the GRD, instead of the minimum growth rate.

\section{APPENDIX C}

\section{The Interference of Growth Rate Increase on Growth Rate Decrease}

In this appendix, we focus on the question why the timing of SPB is locked in February for maximum GRD ranging from October to December in Fig. 2d. We will show that the increasing growth rate from February to March cancels the effect of GRD, because the latter occurs sufficiently close to the former and therefore blocks the shift of SPB.

To illustrate the role of increasing growth rate, we start with the simplest case of a minimum growth rate. We will use a constant growth rate except for a minimum at February (hereafter referred to as the minimum case; Fig. C1a). We increase the amplitudes of the seasonal cycle, or GRD, from a weak amplitude $\delta a=0.1$ to a large amplitude $\delta a=0.9$ for both the strong and weak damping cases $(\bar{a}=0.7$ and 0.9$)$. In the case of strong damping $\bar{a}=0.7$ (black squares in Fig. C1c), the SPB timings are always phase locked to February when $\delta a$ is larger than 0.5 . For the weak damping cases ( $\bar{a}$ increases to 0.9 ; black triangles in Fig. C1c), the timing of the SPB is also locked to March, with a slight delay of one month. This lock of SPB month with increasing seasonal cycle occurs because the GRD effect (from January to February) on decreasing SNR is canceled by the immediate strong increase of growth rate (from February to March). To see this more clearly, we perform another set of experiments with a maximum in February (the maximum case, Fig. C1b). In contrast to the minimum cases in Fig. C1a, in the case of weak damping $\bar{a}=0.9$, when $\delta a$ increases, the timing of SPB is shifted later almost linearly (blue triangles in Fig. C1c). The shift of the SPB is caused by the increased GRD magnitude, with no cancelation effect from the growth rate increase immediately afterward. In comparison with the strong damping case, the SPB is delayed by more than two months (blue squares vs blue triangles in Fig. C1c). Therefore, for weak damping (large $\bar{a}$ ), the timing of the SPB will be delayed more from the GRD and this delay increases with the amplitude of the seasonal cycle (amplitude of GRD). Therefore, in the absence of the increasing growth rate (the maximum case), the SPB timing is no longer phase locked in March, at least for the weak damping case $\bar{a}=0.9$. The comparison of the maximum case with the minimum case suggests that it is the increasing growth rate immediately after the GRD that cancels the GRD effect and in turn blocks the shift of SPB with increasing GRD magnitude.

The role of increasing growth rate in blocking the SPB from occurring after February is further studied in Fig. C2, in which an increasing growth rate $r$ is imposed immediately after the GFD (January-February) from February to March with increasing amplitude in the case of weak damping $(\bar{a}=0.9)$. For a weak $r=0.02$, the SPB occurs three months after the GRD in May. When $r$ increases to 0.06 and then 0.2, the SPB shifts earlier from May to April and then to March (Figs. C2b and A2c). Figure C2d further shows that when $r$ increases to 0.1 and higher, the SPB timing will be blocked to March. This case can be used to understand the large $\bar{a}$ case in Liu et al. (2019). In that case, the timing of SPB appears to occur in the period of increasing growth rate (their Fig. 3b). Our study suggests that, however, this concurrence of SPB and increasing growth rate does not mean that the SPB is driven by the increasing growth rate. Instead, the SPB is caused more by the GRD, and is then delayed as the system has a weak damping.

Now we can further understand why the timing of SPB is locked to February for weak damping (large $\bar{a}$ ) cases in Fig. 2d. For weak damping, the SPB month will lag the time of the maximum GRD and occurs after February when the timing of 
(a) Minimum case

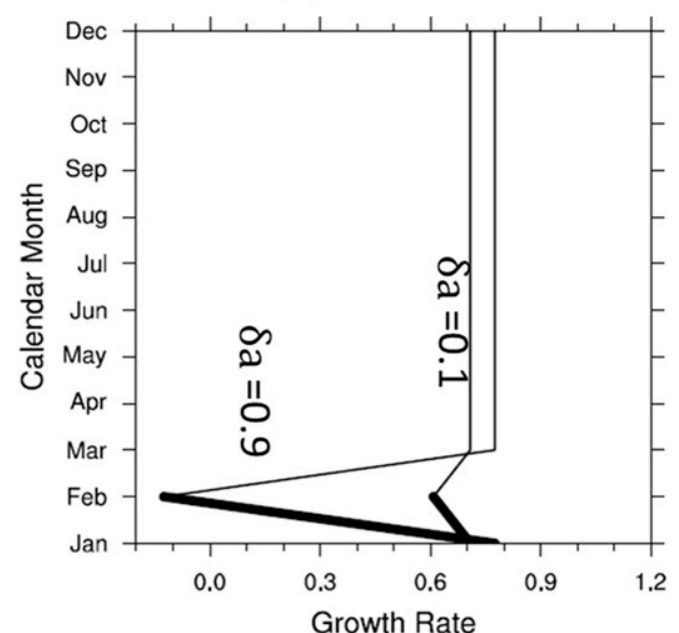

(b) Maximum case

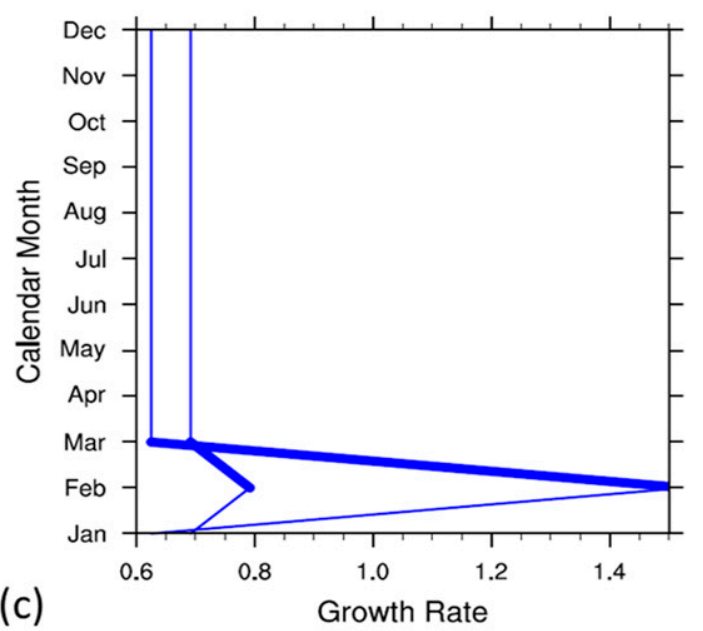

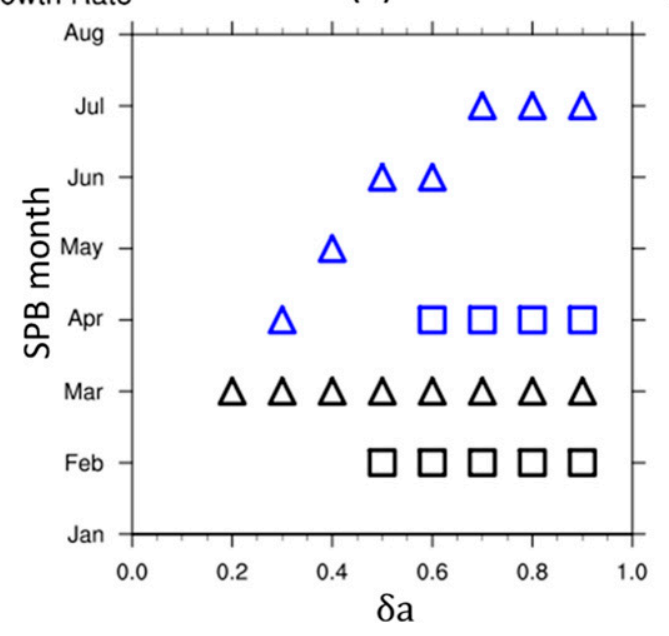

FIG. C1. The (a) minimum and (b) maximum growth rate in February. The $\delta a$ ranges from 0.1 to 0.9. (c) SPB timing (black) forced by the minimum case [in (a)] while blue indicates the SPB timing forced by the maximum case [in (b)]; squares are the results forced by $\bar{a}=0.7$ while triangles indicate the $\bar{a}=0.9$ case.

the maximum GRD ranges from September to December. On the other hand, there is a large increasing growth rate from February to March, which prevents the phase shift of the SPB after March if the increasing growth rate occurs after the GRD sufficiently close. As such, the SPB is blocked in February. When the timing of maximum GRD ranges from March to August, the SPB month changes with the GRD because the increasing growth rate is far away from the GRD. We also test the effect of the amplitude of the seasonal cycle (i.e., $\delta a$ ) on the SPB for weak damping cases (large $\bar{a})$. When we decrease $\delta a$ from 0.9 to 0.2 (black triangle in Fig. 2d), now 1) the SPB month is phase locked to February when the maximum GRD time ranges from October to December, and 2) the SPB months are closer to the maximum GRD time compared to the cases of lager $\delta a=0.9$. This confirms that a large amplitude of seasonal cycle $\delta a$ will also delay the timing the SPB, qualitatively consistent with the harmonic solution of Liu et al. (2019, their Figs. 3a,b).

\section{APPENDIX D}

\section{Comparison of Growth Rate in the Langevin Equation and AR(1) Model}

The Langevin equation with the incorporation of a seasonal cycle of growth rate is

$$
\frac{d T}{d t}=-b(t) T+N(t)
$$

For Eq. (D.1), the growth rate is $-b$, and the seasonal Langevin equation Eq. (D.1) is discretized in the time step of one month as

$$
X_{t+1}=\left(1-b_{m}\right) X_{t}+N_{t+1} .
$$

Comparing Eqs. (2.1) with (D.2), we find that

$$
-b_{m}=a_{m}-1
$$




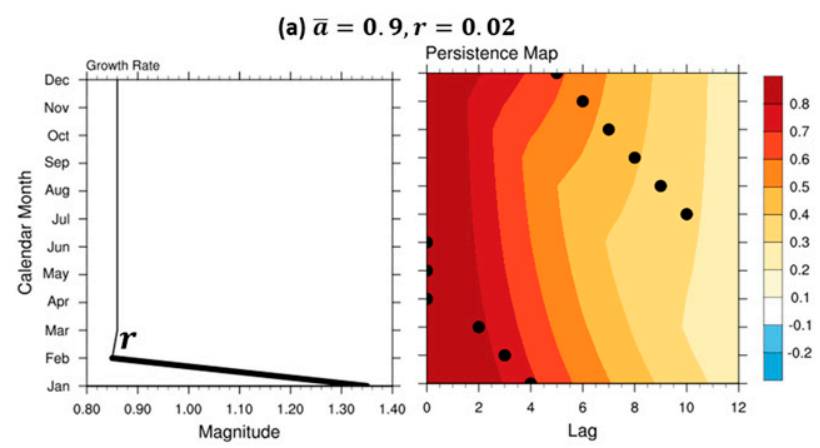

(c) $\bar{a}=0.9, r=0.2$

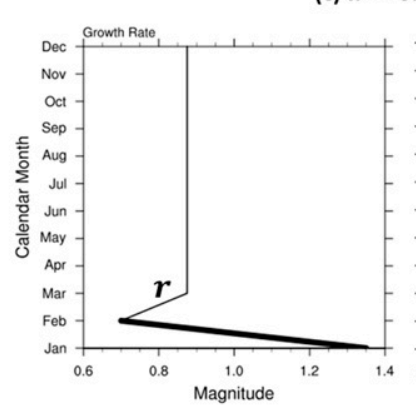

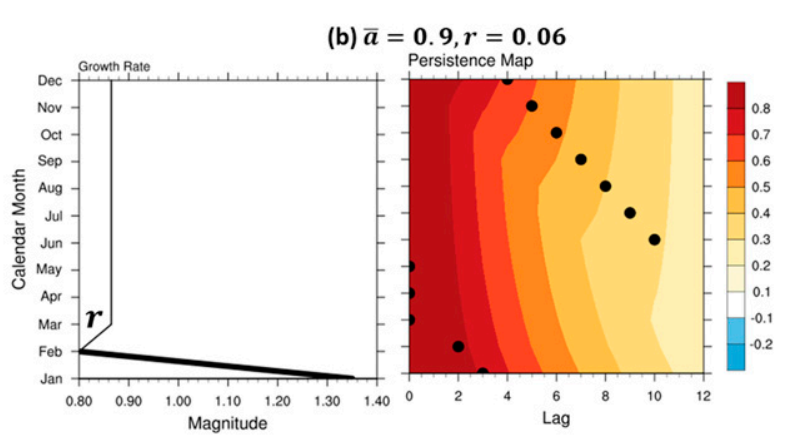

(d)

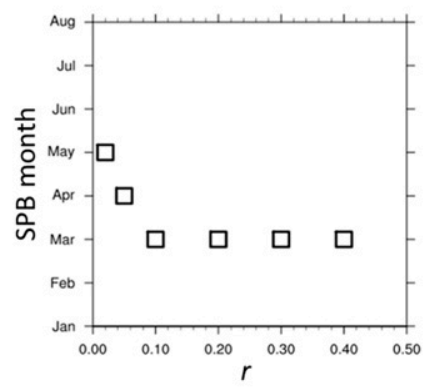

FIG. C2. (a) The left panel is seasonal growth rate; $r$ means the growing growth rate from February to March. The right panel is the persistence map forced by this seasonal cycle. (b),(c) As in (a), but for different seasonal growth rate cases. (d) The black square is the SPB month for different $r$.

In this paper, $a_{m}$ is the growth rate for the AR(1) model. This relationship also fits well between the recharge oscillator model and AR(1) model.

\section{REFERENCES}

Alexander, M. A., C. Deser, and M. S. Timlin, 1999: The reemergence of SST anomalies in the North Pacific Ocean. J. Climate, 12, 2419-2433, https://doi.org/10.1175/1520-0442(1999) 012<2419:TROSAI > 2.0.CO;2.

Chen, D., and M. A. Cane, 2008: El Niño prediction and predictability. J. Comput. Phys., 227, 3625-3640, https://doi.org/ 10.1016/j.jcp.2007.05.014.

_ - _ A. Kaplan, S. E. Zebiak, and D. Huang, 2004: Predictability of El Niño over the past 148 years. Nature, 428, 733-736, https://doi.org/10.1038/nature02439.

Chen, H.-C., and F.-F. Jin, 2020: Fundamental behavior of ENSO phase locking. J. Climate, 33, 1953-1968, https://doi.org/ 10.1175/JCLI-D-19-0264.1.

Goswami, B., and J. Shukla, 1991: Predictability of a coupled ocean-atmosphere model. J. Climate, 4, 3-22, https://doi.org/ 10.1175/1520-0442(1991)004<0003:POACOA > 2.0.CO;2.

Hasselmann, K., 1976: Stochastic climate models. Part I. Theory. Tellus, 28, 473-485, https://doi.org/10.1111/j.2153-3490.1976.tb00696.x.

Jin, E. K., and Coauthors, 2008: Current status of ENSO prediction skill in coupled ocean-atmosphere models. Climate Dyn., 31, 647-664, https://doi.org/10.1007/s00382-008-0397-3.

Jin, F., S. T. Kim, and L. Bejarano, 2006: A coupled-stability index of ENSO. Geophys. Res. Lett., 33, L23708, https://doi.org/ 10.1029/2006GL027221.

Jin, Y., Z. Liu, and X. Rong, 2019a: General seasonal phase-locking of variance and persistence: Application to tropical Pacific,
North Pacific and global ocean. Climate Dyn., 53, 2825-2842, https://doi.org/10.1007/s00382-019-04659-7.

,-- Z. Lu, and C. He, 2019b: Seasonal cycle of background in the tropical Pacific as a cause of ENSO spring persistence barrier. Geophys. Res. Lett., 46, 13 371-13 378, https://doi.org/ 10.1029/2019GL085205.

Kim, S. T., and F.-F. Jin, 2011: An ENSO stability analysis. Part II: Results from the twentieth and twenty-first century simulations of the CMIP3 models. Climate Dyn., 36, 1609-1627, https://doi.org/10.1007/s00382-010-0872-5.

Levine, A. F. Z., and M. J. McPhaden, 2015: The annual cycle in ENSO growth rate as a cause of the spring predictability barrier. Geophys. Res. Lett., 42, 5034-5041, https://doi.org/ 10.1002/2015GL064309.

Liu, Z., Y. Jin, and X. Rong, 2019: A theory for the seasonal predictability barrier: Threshold, timing, and intensity. J. Climate, 32, 423-443, https://doi.org/10.1175/JCLI-D-18-0383.1.

Lu, Z., and Z. Liu, 2018: Orbital modulation of ENSO seasonal phase locking. Climate Dyn., 52, 4329-4350, https://doi.org/ 10.1007/s00382-018-4382-1.

Lübbecke, J. F., and M. J. McPhaden, 2014: Assessing the twentyfirst-century shift in ENSO variability in terms of the Bjerknes stability index. J. Climate, 27, 2577-2587, https://oi.org/ 10.1175/JCLI-D-13-00438.1.

Moon, W., and J. S. Wettlaufer, 2017: A unified nonlinear stochastic time series analysis for climate science. Sci. Rep., 7, 44228, https://doi.org/10.1038/srep44228.

$\mathrm{Mu}$, M., W. Duan, and B. Wang, 2007: Season-dependent dynamics of nonlinear optimal error growth and El NiñoSouthern Oscillation predictability in a theoretical model. J. Geophys. Res., 112, D10113, https://doi.org/10.1029/2005 JD006981. 
Penland, C., and P. Sardeshmukh, 1995: The optimal growth of tropical sea surface temperature anomalies. J. Climate, $\mathbf{8}$, 1999-2024, https://doi.org/10.1175/1520-0442(1995)008<1999: TOGOT $>2.0 . \mathrm{CO} ; 2$.

Rayner, N. A., D. E. Parker, E. B.Horton C. K.Folland, L. V.Alexander, D. P.Rowell, E. C.Kent, and A.Kaplan, 2003: Global analyses of sea surface temperature, sea ice, and night marine air temperature since the late nineteenth century. J. Geophys. Res., 108, 4407, https://doi.org/10.1029/2002JD002670.

Ren, H. L., F. F. Jin, B. Tian, and A. A. Scaife, 2016: Distinct persistence barriers in two types of ENSO. Geophys. Res. Lett., 43, 10 973-10 979, https://doi.org/10.1002/2016GL071015.

Stein, K., N. Schneider, A. Timmermann, and F.-F. Jin, 2010: Seasonal synchronization of ENSO events in a linear stochastic model. J. Climate, 23, 5629-5643, https://doi.org/ 10.1175/2010JCLI3292.1.

Thompson, C., and D. Battisti, 2000: A linear stochastic dynamical model of ENSO. Part I: Model development. J. Climate, 13, 2818-2832, https://doi.org/10.1175/1520-0442(2000)013<2818: ALSDMO $>2.0 . \mathrm{CO} ; 2$.

Torrence, C., and P. J. Webster, 1998: The annual cycle of persistence in the El Nño/Southern Oscillation. Quart. J. Roy. Meteor. Soc., 124, 1985-2004, https://doi.org/10.1002/QJ.49712455010.

Troup, A. J., 1965: The "southern oscillation." Quart. J. Roy. Meteor. Soc., 91, 490-506, https://doi.org/10.1002/qj.49709139009.
Walker, G. T., and E. W. Bliss, 1932: World weather V. Mem. Roy. Meteor. Soc, 4, 53-84.

Webster, P. J., 1995: The annual cycle and the predictability of the tropical coupled ocean-atmosphere system. Meteor. Atmos. Phys., 56, 33-55, https://doi.org/10.1007/BF01022520.

— active systems. Quart. J. Roy. Meteor. Soc., 118, 877-926, https://doi.org/10.1002/qj.49711850705.

Wright, P. B., 1979: Persistence of rainfall anomalies in the central Pacific. Nature, 277, 371-374, https://doi.org/10.1038/277371a0.

Xue, Y., M. A. Cane, S. E. Zebiak, and M. B. Blumenthal, 1994: On the prediction of ENSO: A study with a low-order Markov model. Tellus, 46A, 512-528, https://doi.org/10.3402/tellusa.v46i4. 15641.

Yeh, S.-W., J.-G. Jhun, I.-S. Kang, and B. P. Kirtman, 2004: The decadal ENSO variability in a hybrid coupled model. J. Climate, 17, 1225-1238, https://doi.org/10.1175/1520-0442(2004) 017<1225:TDEVIA > 2.0.CO;2.

Zebiak, S. E., 1989: On the 30-60 day oscillation and the prediction of El Niño. J. Climate, 2, 1381-1387, https://doi.org/10.1175/ 1520-0442(1989)002<1381:OTDOAT >2.0.CO;2.

Zhao, X., J. Li, and W. Zhang, 2012: Summer persistence barrier of sea surface temperature anomalies in the central western North Pacific. Adv. Atmos. Sci., 29, 1159-1173, https://doi.org/ 10.1007/s00376-012-1253-2. 